Dear Author,

Please, note that changes made to the HTML content will be added to the article before publication, but are not reflected in this PDF.

Note also that this file should not be used for submitting corrections. 


\title{
Level-set simulations of buoyancy-driven motion of single and multiple bubbles
}

\author{
Néstor Balcázar ${ }^{\mathrm{a}, *}$, Oriol Lehmkuhl ${ }^{\mathrm{a}, \mathrm{b}}$, Lluís Jofre ${ }^{\mathrm{a}}$, Assensi Oliva ${ }^{\mathrm{a}, *}$ \\ ${ }^{a}$ Heat and Mass Transfer Technological Center (CTTC), Universitat Politècnica de Catalunya - BarcelonaTech (UPC) ETSEIAT, Colom 11, 08222 Terrassa (Barcelona), Spain \\ ${ }^{\mathrm{b}}$ Termo Fluids, S.L., Avda Jacquard 97 1-E, 08222 Terrassa (Barcelona), Spain
}

\section{A R T I C L E I N F O}

\section{Article history:}

Received 15 December 2014

Received in revised form 11 May 2015

Accepted 7 July 2015

Available online $\mathrm{xxxx}$

\section{Keywords:}

Bubble dynamics

Level-set method

Multiphase flow

\begin{abstract}
A B S T R A C T
This paper presents a numerical study of buoyancy-driven motion of single and multiple bubbles by means of the conservative level-set method. First, an extensive study of the hydrodynamics of single bubbles rising in a quiescent liquid is performed, including its shape, terminal velocity, drag coefficients and wake patterns. These results are validated against experimental and numerical data well established in the scientific literature. Then, a further study on the interaction of two spherical and ellipsoidal bubbles is performed for different orientation angles. Finally, the interaction of multiple bubbles is explored in a periodic vertical channel. The results show that the conservative level-set approach can be used for accurate modelling of bubble dynamics. Moreover, it is demonstrated that the present method is numerically stable for a wide range of Morton and Reynolds numbers.
\end{abstract}

(c) 2015 Published by Elsevier Inc.

\section{Introduction}

Bubbles play an important role in many natural and industrial processes. Steam generators in nuclear plants, unit operations in chemical engineering such as distillation, absorption, extraction, heterogeneous catalysis and bubble reactors are only a few among a multitude of applications that involve the motion of bubbles and droplets (Mudde, 2005). These applications have motivated a large number of numerical and experimental investigations of bubble dynamics (see Tables 1 and 2). Despite those efforts, the current understanding of such flows and their predictive models are far from satisfactory, and many challenging problems still remain as pointed out in recent reviews (Mudde, 2005; Tryggvason et al., 2013).

The development of computers has promoted Direct Numerical Simulation (DNS) of the Navier-Stokes equations as another means of performing controlled experiments (Tryggvason et al., 2001, 2013), providing a good way to non-invasive measure of droplet and bubble flows, although computationally demanding. As further advantages, DNS allows us to control the size distribution of bubbles or droplets, their deformability, whether they coalesce or not, and modify the flow conditions (Tryggvason et al., 2013). There are multiple methods for DNS of bubbly flows, for instance: the

\footnotetext{
* Corresponding authors. Fax: +34 937398920

E-mail addresses: nestor@cttc.upc.edu (N.Balcázar),termofluids@termofluids.com (O. Lehmkuhl),cttc@cttc.upc.edu, cttc@upc.edu (A. Oliva).
}

front tracking (FT) method (Unverdi and Tryggvason, 1992; Tryggvason et al., 2001), level set (LS) methods (Osher and Sethian, 1988; Sussman et al., 1994; Olsson and Kreiss, 2005; Sheu et al., 2009; Balcázar et al., 2014), volume-of-fluid (VOF) methods (Hirt and Nichols, 1981; Van Sint Annaland et al., 2005), and hybrid VOF/LS methods (Sussman and Puckett, 2000). In these methods, two-phase flow is treated as a single flow with the density and viscosity varying smoothly across the moving interface which is captured in an Eulerian framework (VOF, LS, VOF/LS) or in a Lagrangian framework (FT). Although the idea behind these methods is similar, their numerical implementation may differ greatly.

In Front-tracking methods (Unverdi and Tryggvason, 1992; Tryggvason et al., 2001) a stationary Eulerian grid is used to solve the fluid flow, while the interface is tracked explicitly by a separate Lagrangian grid. This method is extremely accurate but also rather complex to implement due to the fact that dynamic re-meshing of the Lagrangian interface mesh is required (Deen et al., 2009). In VOF methods (Hirt and Nichols, 1981; Van Sint Annaland et al., 2005), the interface is given implicitly by a color function, defined to be the fraction of volume within each cell of one of the fluids. In order to advect the VOF function, the interface needs to be reconstructed by using a geometric technique. An advantage of VOF method is the fact that accurate algorithms can be used to advect the interface, so that the mass can be conserved, while still maintaining a sharp representation of the interface. However a disadvantage of the VOF method is the fact that it is difficult to compute accurate curvatures from the color function, because it 
Table 1

Some experimental works on the motion of single and multiple bubbles.

\begin{tabular}{|c|c|}
\hline Reference (year) & System studied \\
\hline Hnat and Buckmaster (1976) & Spherical cap and skirted bubbles \\
\hline Clift et al. (1978) & Bubbles and droplets \\
\hline \multirow[t]{2}{*}{ Bhaga and Weber (1981) } & Single bubbles \\
\hline & Coalescence of two bubbles \\
\hline Duineveld (1998) & $\begin{array}{l}\text { Interaction and coalescence of two } \\
\text { bubbles }\end{array}$ \\
\hline Sanada et al. (2009) & $\begin{array}{l}\text { Interaction and coalescence of two } \\
\text { bubbles }\end{array}$ \\
\hline $\begin{array}{l}\text { Takemura and Magnaudet } \\
\text { (2003) }\end{array}$ & Bubble-wall interactions \\
\hline Stewart (1995) & Rising motion of bubble swarms \\
\hline Zenit et al. (2001) & $\begin{array}{l}\text { Suspension of bubbles in a vertical } \\
\text { channel }\end{array}$ \\
\hline Martínez-Mercado et al. (2007) & Mono-disperse bubbly flow \\
\hline
\end{tabular}

presents a step discontinuity at the interface. In level-set (LS) methods (Osher and Sethian, 1988; Sussman et al., 1994) the interface is represented by the zero-contour of a smooth signed distance function. The evolution of this function in space and time is governed by an advection equation, combined with a special re-distancing algorithm. One of the advantage of level-set approach is the fact that interface curvatures and normals can be accurately computed. However, a disadvantage of this method is that the discrete solution of transport equations is prone to numerical error and it leads to loss or gain of mass. On the basis of the aforementioned advantages and disadvantages of the VOF and LS methods, it can be found that they are complementary, therefore coupled VOF-LS approaches have been proposed in Sussman and Puckett (2000), Wang et al. (2009) and Sun and Tao (2010). In these methods interface properties are obtained through a level-set method while mass conservation problems are avoided by using the VOF method. Although these methods extracts the advantages

\section{Table 2}

Some numerical works that report simulations of single and multiple bubbles. FT (front tracking), VOF (volume-of-fluid), CLSVOF (coupled level-set and volume-of-fluid), BFT (boundary-fitted mesh), MP (moving particle semi-implicit), LS (level-set), LB (lattice-Boltzmann).

\begin{tabular}{|c|c|c|}
\hline Reference (year) & Method & System studied \\
\hline Ryskin and Leal (1984) & BFM & Single bubbles \\
\hline Van Sint Annaland et al. (2005) & VOF & $\begin{array}{l}\text { Single bubbles } \\
\text { Coalescence of two bubbles }\end{array}$ \\
\hline Hua et al. (2008) & FT & $\begin{array}{l}\text { Single bubbles } \\
\text { Coalescence of two bubbles }\end{array}$ \\
\hline Ohta et al. (2010) & CLSVOF & Single bubbles \\
\hline Ohta and Sussman (2012) & CLSVOF & Single bubbles \\
\hline Chen et al. (2011) & MP & Coalescence of two bubbles \\
\hline Smolianski et al. (2008) & LS & $\begin{array}{l}\text { Single bubbles } \\
\text { Multiple bubble interaction }\end{array}$ \\
\hline Gupta and Kumar (2008) & LB & $\begin{array}{l}\text { Single bubbles } \\
\text { Multiple bubble interaction }\end{array}$ \\
\hline Cheng et al. (2010) & LB & $\begin{array}{l}\text { Single bubbles } \\
\text { Coalescence of two bubbles } \\
\text { Multiple bubble interaction }\end{array}$ \\
\hline Yu et al. (2011) & LB & $\begin{array}{l}\text { Single bubbles } \\
\text { Interaction of two bubbles } \\
\text { Multiple bubble interaction }\end{array}$ \\
\hline Liu and Valocchi (2012) & LB & Single bubbles \\
\hline Chen et al. (1998) & VOF & Coalescence of two bubbles \\
\hline Hasan and Zakaria (2011) & VOF & Coalescence of two bubbles \\
\hline Legendre and Magnaudet (2003) & BFM & Interaction of two bubbles \\
\hline Hallez and Legendre (2011) & BFM & Interaction of two bubbles \\
\hline Pournader and Mortazavi (2013) & FT & Interaction of two drops \\
\hline Chakraborty et al. (2013) & CLSVOF & $\begin{array}{l}\text { Single bubbles } \\
\text { Coalescence of two bubbles }\end{array}$ \\
\hline Bunner and Tryggvason (2002) & FT & Homogeneous bubbly flows \\
\hline Bunner and Tryggvason (2003) & FT & Homogeneous bubbly flows \\
\hline Esmaeeli and Tryggvason (2005) & FT & Homogeneous bubbly flows \\
\hline
\end{tabular}

of VOF and LS methods, they are more complicated than VOF or LS method. Recently, a conservative level-set method (CLS) has been introduced by Olsson and Kreiss (2005), where a regularized indicator function has been employed in place of the signed distance function. In addition, Balcázar et al. $(2014,2015)$ have generalized the CLS method to compute two-phase flows with surface tension effects in the framework of collocated unstructured meshes using a finite-volume approach. An advantage of CLS method is that mass conservation problem associated to standard formulations of level-set methods is greatly reduced. Additionally this approach benefits of automatic handling of topology changes and efficient parallelization with no additional cost.

Some experimental and numerical studies on the buoyancy-driven motion of single and multiple bubbles have been summarized in Tables 1 and 2 respectively. To the best of the author's knowledge there are not detailed computations of bubble dynamics using the conservative level-set (CLS) method (Olsson and Kreiss, 2005; Balcázar et al., 2014). Therefore, in order to contribute in the understanding of the implications introduced by the different frameworks used for interface capturing, this paper presents an extensive validation and verification of the CLS method (Balcázar et al., 2014). In addition, due to the limited number of numerical studies on the interaction of multiple bubbles using interface capturing methods (VOF, LS, VOF/LS), this work presents detailed simulations of bubble interactions in the context of the CLS method. These numerical findings are compared with experimental and numerical results from the literature, some of them have been described in Tables 1 and 2. Thus, the objectives of this research are as follows: first to demonstrate the accuracy of the CLS method to predict bubble shapes, drag coefficients and wake patterns of single bubbles for various flow regimes; second, to investigate hydrodynamic interaction mechanisms of bubble pairs for different configuration angles; and, the third objective is to apply the CLS method to simulate the buoyancy-rising motion of a set of bubbles in a vertical channel.

The present paper is organized as follows: The governing equations employed in this study are given in Section 2. Section 3 is devoted to the description of the numerical method. Simulation results for single and multiple bubbles are presented in Section 4. Finally, concluding remarks are given in Section 5.

\section{Mathematical formulation}

The conservation of momentum and mass of two immiscible incompressible and Newtonian fluids is described by the Navier-Stokes equations defined on a spatial domain $\Omega$ with boundary $\partial \Omega$ :

$$
\begin{aligned}
& \frac{\partial}{\partial t}\left(\rho_{k} \mathbf{v}_{k}\right)+\nabla \cdot\left(\rho_{k} \mathbf{v}_{k} \mathbf{v}_{k}\right)=\nabla \cdot \mathbf{S}_{k}+\rho_{k} \mathbf{g} \text { in } \Omega_{k} \\
& \mathbf{S}_{k}=-p_{k} \mathbf{I}+\mu_{k}\left(\nabla \mathbf{v}_{k}+\left(\nabla \mathbf{v}_{k}\right)^{T}\right) \\
& \nabla \cdot \mathbf{v}_{k}=0 \text { in } \Omega_{k}
\end{aligned}
$$

Here, $\Omega=\Omega_{1} \cup \Omega_{2} \cup \Gamma, k=\{1,2\}$ denote the subdomains associated with the two different fluid phases, $\Gamma=\partial \Omega_{1} \cap \partial \Omega_{2}$ is the fluid interface, $\rho$ and $\mu$ denote the density and dynamic viscosity of the fluids, $\mathbf{v}$ is the velocity field, $\mathbf{g}$ is the gravity acceleration, $p$ is the pressure, $\mathbf{S}$ is the stress tensor and $\mathbf{I}$ is the identity tensor. Assuming no mass transfer between the fluids yields a continuous velocity condition at the interface:

$\mathbf{v}_{1}=\mathbf{v}_{2}$ in $\Gamma$

The jump in normal stresses along the fluid interface is balanced by the surface tension. Neglecting the variations of the surface tension coefficient $\sigma$ gives the following boundary condition for momentum conservation at the interface: 
$\left(\mathbf{S}_{1}-\mathbf{S}_{2}\right) \cdot \mathbf{n}=\sigma \kappa \mathbf{n} \quad$ in $\Gamma$

where $\mathbf{n}$ is the unit normal vector outward to $\partial \Omega_{1}$ and $\kappa$ is the interface curvature. Eqs. (1)-(3) and Eqs. (4) and (5) can be combined into a set of equations for a single fluid in $\Omega$, with a singular source term for the surface tension force at the interface $\Gamma$ (Peskin, 1977; Brackbill et al., 1992):

$\frac{\partial}{\partial t}(\rho \mathbf{v})+\nabla \cdot(\rho \mathbf{v v})=-\nabla p+\nabla \cdot \mu\left(\nabla \mathbf{v}+(\nabla \mathbf{v})^{T}\right)+\left(\rho-\rho_{0}\right) \mathbf{g}+\sigma \kappa \mathbf{n} \delta_{\Gamma}$

$\nabla \cdot \mathbf{v}=0$

where $\mathbf{v}$ is the velocity vector, $p$ is the pressure, $\mathbf{g}$ is the gravity acceleration, $\delta_{\Gamma}$ is the Dirac delta function concentrated at the interface, $\sigma$ is the constant surface tension coefficient, $\kappa$ is the curvature of the interface, and $\mathbf{n}$ denotes the unit normal vector on the interface. Since $\rho$ and $\mu$ are constant in each fluid with a jump discontinuity at the interface, they can be defined as:

$\rho=\rho_{1} H_{1}+\rho_{2}\left(1-H_{1}\right) \quad \mu=\mu_{1} H_{1}+\mu_{2}\left(1-H_{1}\right)$

Here the subscripts 1 and 2 denote fluid 1 and fluid 2, respectively, while $H_{1}$ is the Heaviside step function that is one at fluid 1 and zero elsewhere. However, in order to avoid numerical instabilities at the interface, a continuous treatment of physical properties is adopted in present work, according to Eq. (17), introduced in the context of the CLS method. Present simulations are performed on a periodic domain, hence, an additional body force was added to Eq. (6) to adjust the pressure gradient such that the flow rate of the fluid phase is exactly zero (Yin and Koch, 2008). Following the work of Bunner and Tryggvason (2002) and Yin and Koch (2008), this force is equivalent to the pressure gradient generated by the base of a flow container which prevents the gravitational force to cause the acceleration of the entire flow field in the downward vertical direction. Indeed, a force equal to the space-averaged density times the gravitational acceleration, $\rho_{0} \mathbf{g}$ where $\rho_{0}=$ $\left(V_{\Omega}\right)^{-1} \int_{\Omega}\left(\left.H_{1}\right|_{t=0} \rho_{1}+\left(1-\left.H_{1}\right|_{t=0}\right) \rho_{2}\right) d V$, is subtracted from the right hand side of the momentum equation.

The two major challenges of simulating interfaces between different fluids are to maintain a sharp front and to compute the surface tension accurately (Tryggvason et al., 2001). Regarding the first issue, the CLS method implemented in Balcázar et al. (2014) has been selected for interface capturing. In this method the interface is implicitly represented by a regularized indicator function, $\phi$ :

$\phi(\mathbf{x}, t)=\frac{1}{2}\left(\tanh \left(\frac{d(\mathbf{x}, t)}{2 \varepsilon}\right)+1\right)$

where $\varepsilon$ is a parameter that sets the thickness of the profile. With this profile the interface $\Gamma$ is defined by the location of the $\phi=0.5$ iso-surface, $\Gamma=\{\mathbf{x} \mid \phi(\mathbf{x}, t)=0.5\}$. by the solution of Eq. (6). The interface transport equation can be written in conservative form provided the velocity field is solenoidal, $\nabla \cdot \mathbf{v}=0$, namely,

$\frac{\partial \phi}{\partial t}+\nabla \cdot \phi \mathbf{v}=0$

Furthermore, an additional re-initialization equation is introduced to keep the profile and thickness of the interface sharp and constant (Olsson and Kreiss, 2005; Balcázar et al., 2014),

$\frac{\partial \phi}{\partial \tau}+\nabla \cdot \phi(1-\phi) \mathbf{n}=\nabla \cdot \varepsilon \nabla \phi$

This equation is advanced in pseudo-time $\tau$, it consists of a compressive term, $\left.\phi(1-\phi) \mathbf{n}\right|_{\tau=0}$, which forces the level-set function to be compressed onto the interface along the normal vector $\mathbf{n}$, and
The CLS function $\phi$ is advected by a vector field $\mathbf{v}$ which is given of a diffusion term $\nabla \cdot \varepsilon \nabla \phi$ that ensure the profile remains of characteristic thickness $\varepsilon$.

Implementing surface tension in a numerical scheme involves two issues: the curvature $\kappa$ needs to be determined and the resulting pressure jump must be applied appropriately to the fluids. The aforementioned problems can be conveniently addressed through the CSF method (Brackbill et al., 1992). Thus, the singular term, $\sigma \kappa \mathbf{n} \delta_{\Gamma}$, is converted to a volume force as follows,

$\sigma \kappa \mathbf{n} \delta_{\Gamma}=\sigma \kappa(\phi) \nabla \phi$

where $\kappa(\phi)$ and $\mathbf{n}$ are given by

$\mathbf{n}=\frac{\nabla \phi}{\|\nabla \phi\|}$

$\kappa(\phi)=-\nabla \cdot \mathbf{n}$

To obtain a cell averaged value, the curvature is integrated over each finite volume $\Omega_{P}$ :

$\kappa_{P}=-\frac{1}{V_{P}} \int_{\Omega_{P}} \nabla \cdot \mathbf{n} d V$

Applying the Gauss theorem yields

$\kappa_{P}=-\frac{1}{V_{P}} \int_{S_{P}} \mathbf{n} \cdot d \mathbf{A}$

Here $\nabla \phi$ is calculated by using the least-squares method (Kothe et al., 1996; Balcázar et al., 2014), $\mathbf{A}$ is the area vector, $V_{P}$ is the volume and $S_{P}$ is the surface of $\Omega_{P}$. The reader is referred to Balcázar et al. (2014) for further details on the application of the least squares method for gradient evaluation. In addition, the fluid properties are regularized by employing the level-set function,

$\rho=\rho_{1} \phi+\rho_{2}(1-\phi) \quad \mu=\mu_{1} \phi+\mu_{2}(1-\phi)$

\section{Numerical method}

Following the work of Balcázar et al. (2014), the Navier-Stokes and level-set equations have been discretized on a collocated unstructured grid arrangement by means of the finite-volume method. In order to avoid unphysical oscillations at the discontinuities, a TVD Superbee limiter is used to discretize the convective term of interface advection Eq. (10); while compressive term of re-initialization Eq. (11) is discretized by CD scheme. Regarding the momentum Eq. (6) a central difference (CD) scheme is used to discretize the convective term unless otherwise stated. Diffusive fluxes at the faces are approximated by CD scheme. A distance-weighted linear interpolation is used to find the face values of physical properties and interface normals, while gradients are computed at cell centroids by using the least-squares method.

The velocity-pressure coupling has been solved by means of a classical fractional step projection method (Chorin, 1968). Thus, momentum Eq. (6) is decomposed into two steps:

$\frac{\rho \mathbf{v}^{*}-\rho \mathbf{v}^{n}}{\Delta t}=-\frac{3}{2} \mathbf{A}_{h}\left(\rho \mathbf{v}^{n}\right)+\frac{1}{2} \mathbf{A}_{h}\left(\rho \mathbf{v}^{n-1}\right)+\mathbf{D}_{h}\left(\mathbf{v}^{n}\right)+\rho \mathbf{g}+\sigma \kappa \nabla_{h}(\phi)$

and

$\mathbf{v}^{n+1}=\mathbf{v}^{*}-\frac{\Delta t}{\rho} \nabla_{h}\left(p^{n+1}\right)$

where $\nabla_{h}$ represents the gradient operator, $D_{h}(\mathbf{v})=\nabla_{h}$ ot $\mu\left(\nabla_{h} \mathbf{v}+\nabla_{h}^{T} \mathbf{v}\right)$ is the diffusion operator, and $\mathbf{A}_{h}(\rho \mathbf{v})=\nabla_{h} \cdot(\rho \mathbf{v v})$ is the convective operator. The resulting velocity $\mathbf{v}^{*}$ from Eq. (18), which does not satisfy the continuity Eq. (7), is corrected by 
Eq. (19). Substituting Eq. (18) into the continuity Eq. (7) yields a Poisson equation for pressure,

$\nabla_{h} \cdot\left(\frac{1}{\rho} \nabla_{h}\left(p^{n+1}\right)\right)=\frac{1}{\Delta t} \nabla_{h} \cdot\left(\mathbf{v}^{*}\right)$

Poisson Eq. (20) discretization leads to a linear system, which is solved by using a preconditioned conjugate gradient method. In order to avoid pressure-velocity decoupling when the pressure projection is made on collocated meshes (Rhie and Chow, 1983; Felten and Lund, 2006), a cell face velocity $\mathbf{v}_{f}$ is defined so that $\nabla_{h} \cdot \mathbf{v}=0$ (see Eq. (7)) in each control volume. Namely in discretized form:

$\mathbf{v}_{f}=\sum_{q \in\{P, F\}} \frac{1}{2}\left(\mathbf{v}_{q}^{n+1}+\frac{\Delta t}{\rho\left(\phi_{q}^{n}\right)}\left(\nabla_{h} p^{n+1}\right)_{q}\right)-\frac{\Delta t}{\rho_{f}}\left(\nabla_{h} p^{n+1}\right)_{f}$

where $P$ and $F$ are denoting the adjacent cell nodes to the face $f$. The reader is referred to Balcázar et al. (2014) for technical details of Eq. (21). This velocity is used to advect the level-set function and momentum in Eqs. (10) and (18) respectively.

For the temporal discretization, explicit Adams-Bashforth scheme is used for the momentum Eq. (18), while for the corrector Eq. (19) an explicit first-order scheme has been used. Advection Eq. (10) and Re-initialization Eq. (11) are integrated in time with a 3-step third-order accurate TVD Runge-Kutta method. Solving Eq. (11) to steady-state results in a smooth transition of $\phi$ at the interface that depends of the diffusion coefficient $\varepsilon$. In this work, numerical simulations were performed by setting $\varepsilon=0.5 \mathrm{~h}$ where $h=\left(V_{P}\right)^{1 / 3}$ is the characteristic size of the grid cell $\Omega_{P}$. Therefore, $\varepsilon$ is chosen to be as small as possible in order to keep a sharp representation of the interface, while numerical stability of Eq. (11) is not affected. In present simulations one iteration per physical time step of re-initialization Eq. (11) was sufficient to keep the profile of the level-set function.

The time increment $\Delta t$, which is limited by the CFL conditions and the stability condition for the capillary force, is given by:

$\Delta t=0.1 \min \left(\frac{h}{|\mathbf{v}|}, \frac{\rho h^{2}}{\mu},\left(\frac{h}{|\mathbf{g}|}\right)^{1 / 2}, h^{3 / 2}\left(\frac{\rho_{1}+\rho_{2}}{4 \pi \sigma}\right)^{1 / 2}\right)$

The global algorithm for solving the equations can be summarized as follows:

1. Calculate $\Delta t$ by Eq. (22).

2. Solve level-set advection Eq. (10).

3. Solve re-initialization Eq. (11) for steady state of the conservative level-set function $\phi$.

4. Calculate $\mathbf{v}$ and $p$ by the fractional-step method: Calculate the predicted velocity by Eq. (18). Solve Eq. (20) for pressure.

Calculate the corrected velocity by Eq. (19).

5. Calculate $\mathbf{v}_{f}$ by Eq. (21).

6. Repeat steps 1-5 until the desired time-level.

The described two-phase flow solver was implemented in an in-house code called TermoFluids (Lehmkuhl et al., 2007), which is a $\mathrm{C}++$ code designed for direct numerical simulation and large eddy simulation of turbulent flows (Trias and Lehmkuhl, 2011). The reader is referred to Balcázar et al. (2014) for technical details of the spatial and temporal discretizations of the Navier-Stokes and level-set equations on collocated unstructured grids.

\section{Results and discussion}

\subsection{Buoyancy-driven motion of a single bubble}

In order to verify and to validate the accuracy of the CLS method, this section is devoted to investigate the buoyancy-driven motion of a single bubble. The bubble shape and its rise velocity, which is correlated to the drag coefficient, is a complex function of the hydrodynamics, viscous and interfacial forces. The experiments of Clift et al. (1978) and Bhaga and Weber (1981), and others provide a fairly detailed picture of the motion of bubbles and drops through a quiescent viscous liquid. The relevant physical quantities in their experiments are: $\rho_{g}, \rho_{l}, \mu_{g}, \mu_{l}, \sigma, d, g, U_{T}$, where $d=(6 \mathrm{~V} / \pi)^{1 / 3}$ is the spherical volume equivalent diameter of the bubble, and $U_{T}$ is the rise velocity. Nondimensionalization results in the next parameters:

$M \equiv \frac{g \mu_{l}^{4} \Delta \rho}{\rho_{l}^{2} \sigma^{3}} \quad E o \equiv \frac{g d^{2} \Delta \rho}{\sigma} \quad \operatorname{Re} \equiv \frac{\rho_{l} U_{T} d}{\mu_{l}} \quad \eta_{\rho} \equiv \frac{\rho_{l}}{\rho_{g}} \quad \eta_{\mu} \equiv \frac{\mu_{l}}{\mu_{g}}$

where $\eta_{\rho}$ and $\eta_{\mu}$ are respectively the density and viscosity ratio; $M$ is the Morton number, $\Delta \rho=\rho_{l}-\rho_{g}$, specifies the density difference between the fluid phases; Eo is the Eötvös number, and $R e$ is the Reynolds number. For given fluids, the Eötvös number is a characteristic of the bubble size and the Morton number is a parameter representing the viscosity of the liquid. For the sake of simplicity, we also introduce the following non-dimensional time, $t^{*}=t \sqrt{\mathrm{g} / \mathrm{d}}$.

The computational setup is schematically indicated in Fig. 1. In the initial state, the spherical bubble is located on the symmetry axis of the cylinder. The numerical simulations were performed on a cylindrical domain of $\left(D_{\Omega}, H_{\Omega}\right)=(8 d, 12 d)$ where $D_{\Omega}$ is the cylinder diameter and $H_{\Omega}$ is the cylinder height (see Fig. 1 ), under periodic boundary conditions between the top and bottom boundaries, and Neumann boundary condition at the side wall. Moreover, with the intend of saving computational resources such as computational time and occupation of memory, the present simulations were computed on a non uniform hexahedral mesh, as show in Fig. 1b. The mesh was generated by a constant step extrusion of a two-dimensional unstructured grid along the symmetry axis of the cylinder, being the step size $H_{\Omega} / N_{\text {planes }}$, where $N_{\text {planes }}$ is the number of planes in which the vertical axis is divided (see Fig. 1b and c). The mesh was concentrated around the symmetry axis of the domain, where a uniform grid size $\left(h_{\min }\right)$ was fixed, to maximize resolution of the bubble. The mesh size grows exponentially to the border, where it reaches a maximum size of $h_{\max } \approx 10 h_{\min }$ (see Fig. 1b). Grid refinement and domain size studies were performed, for the condition Eo $=116, M=41.1, \eta_{\rho}=100$ and $\eta_{\mu}=100$. Fig. 2 a shows the variation in $R e$ as a function of dimensionless time $t^{*}$ and Table 3 shows the value of the computed terminal Reynolds number and the relative error. As the grid size is reduced, the relative difference of $R e$ between successive meshes becomes small (see Fig. 3).

Fig. 2b and Table 4 display results obtained on different domain sizes. A decrease in cylinder diameter reduces the bubble rise velocity $(R e)$, while the shape of the bubble remains almost unchanged. These observations are confirmed by empirical and numerical findings reported by Harmathy (1960), Mukundakrishnan et al. (2007), Hua et al. (2008) and Van Sint Annaland et al. (2005). Therefore, a domain size $\left(D_{\Omega}, H_{\Omega}\right)=(8 d, 12 d)$ and cell size $h=d / 30$ were chosen as the standard conditions for numerical tests unless otherwise stated (see Fig. 1 for a detailed description).

\subsubsection{Terminal velocity and shape}

Fig. 4 shows some representative examples of bubble regimes, in which numerical results are compared against experiments reported by Bhaga and Weber (1981). Generally, small bubbles, which have low Eötvös number $(E o<1)$ tend to maintain the spherical shape, no matter how large the Reynolds number. Similarly, for low Reynolds number $(\operatorname{Re}<1)$, the bubbles will also tend to be spherical, no matter how large the Eötvös number 
(a)

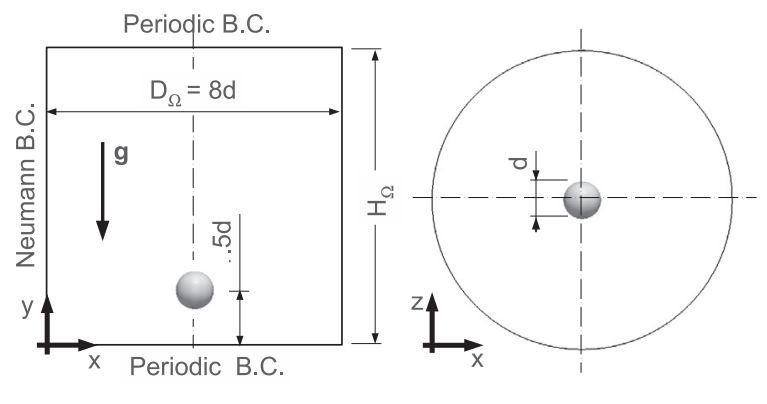

(b)

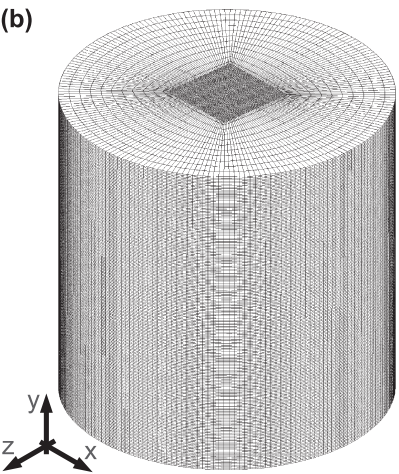

\begin{tabular}{ll}
$\left(\mathrm{D}_{\Omega}, \mathrm{H}_{\Omega}\right)$ & $\mathrm{h}_{\min }$ \\
\hline$(8 \mathrm{~d}, 12 \mathrm{~d})$ & $\mathrm{d} / 20$ \\
$(8 \mathrm{~d}, 12 \mathrm{~d})$ & $\mathrm{d} / 25$ \\
$(8 \mathrm{~d}, 12 \mathrm{~d})$ & $\mathrm{d} / 30$ \\
$(6 \mathrm{~d}, 12 \mathrm{~d})$ & $\mathrm{d} / 30$ \\
$(4 \mathrm{~d}, 12 \mathrm{~d})$ & $\mathrm{d} / 30$ \\
$(8 \mathrm{~d}, 16 \mathrm{~d})$ & $\mathrm{d} / 30$ \\
$(8 \mathrm{~d}, 8 \mathrm{~d})$ & $\mathrm{d} / 60$ \\
\hline
\end{tabular}

Fig. 1. Computational system for single bubble simulations. (a) Sketch of initial condition and boundary conditions. (b) Mesh structure. (c) Mesh configuration.
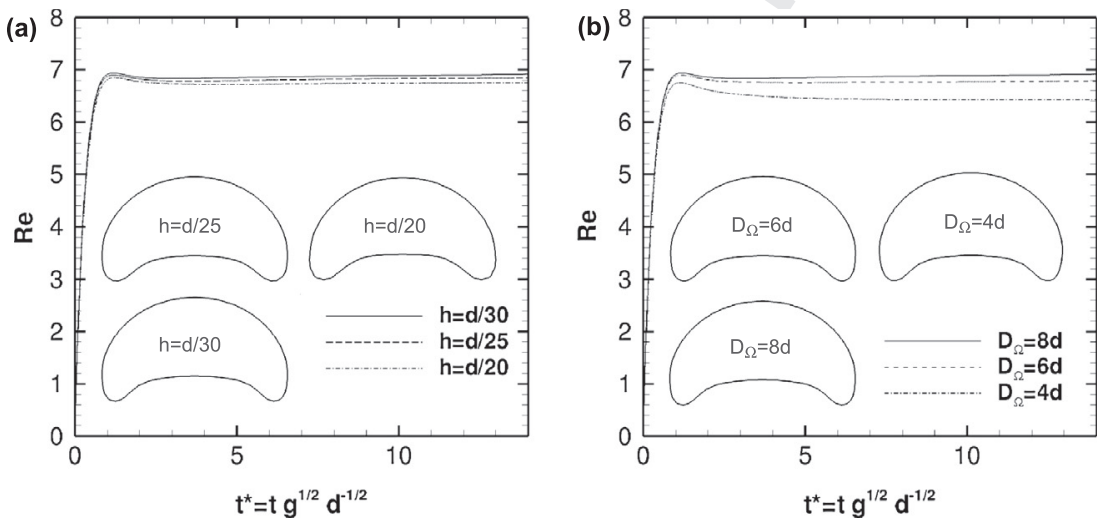

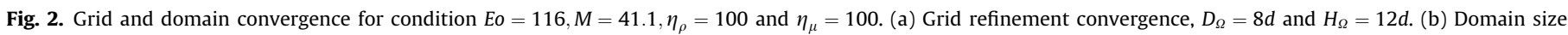
convergence, $h=d / 30$ and $H_{\Omega}=8 d$.

Table 3

Influence of the grid size $(h)$ on the Reynolds number, $E 0=116, M=41.1, \eta_{\rho}=100$ and $\eta_{\mu}=100$. Experimental reference $R e=7.16$ (Bhaga and Weber, 1981). Mesh configuration and domain are shown in Fig. 1.

\begin{tabular}{lllll}
\hline Mesh & Cell size & $\left(D_{\Omega}, H_{\Omega}\right)$ & $R e$ & Relative error $\left(\varepsilon_{r}\right)(\%)$ \\
\hline$M_{1}$ & $h=d / 20$ & $(8 d, 12 d)$ & 6.75 & 5.71 \\
$M_{2}$ & $h=d / 25$ & $(8 d, 12 d)$ & 6.84 & 4.47 \\
$M_{3}$ & $h=d / 30$ & $(8 d, 12 d)$ & 6.94 & 3.03 \\
\hline
\end{tabular}
spite of the difference in shapes, the bubbles rise steadily in a vis cous liquid (high $M$ ) along a rectilinear path. Indeed, the present numerical predictions compare fairly well with the experimental findings of Bhaga and Weber (1981), as shown in Fig. 4. Regarding the terminal Reynolds number (see Table 5 and Fig. 4), the maximum error occurred at creeping flow regime with the lowest terminal Reynolds. Similar observations were reported by Hua et al. (2008) using the front-tracking method (see Table 5).

In addition, local velocity vectors, pressure field and vorticity vectors calculated by using the CLS method are shown in Fig. 1, these results correspond to ellipsoidal and skirted bubbles. Regarding the pressure distribution on the bubble surface, there is a maximum pressure on the top of the bubble, while a minimum pressure is localized on the bottom. The velocity vectors have a maximum magnitude around the symmetric axis of the bubbles. Moreover, it is observed that vorticity vectors takes a maximum magnitude around regions with maximum curvature, which corresponds to equator line for ellipsoidal bubbles, and the base of the skirted bubbles.

Fig. 5a demonstrates the influence of Morton number on the bubble motion at a fixed Eo $=116$. The Morton number varies from 5.51 to 848 . It is observed that the instantaneous Reynolds number 


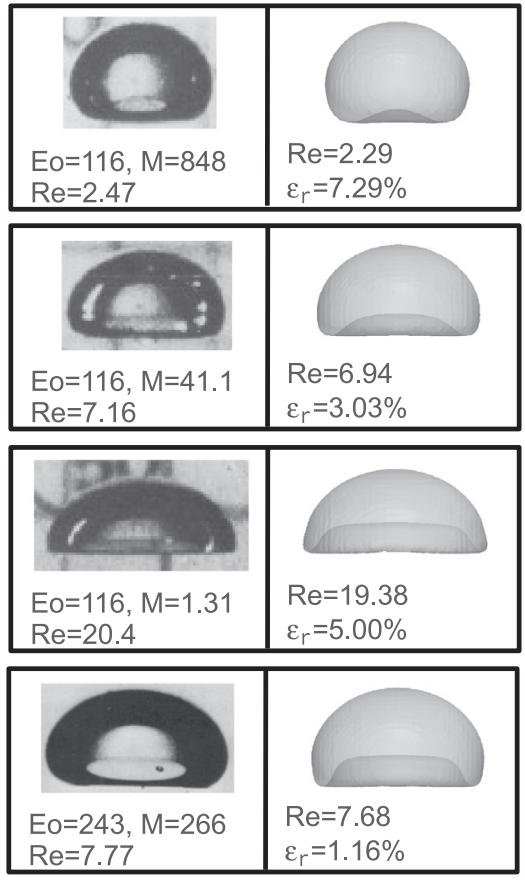

Experiment

CLS method
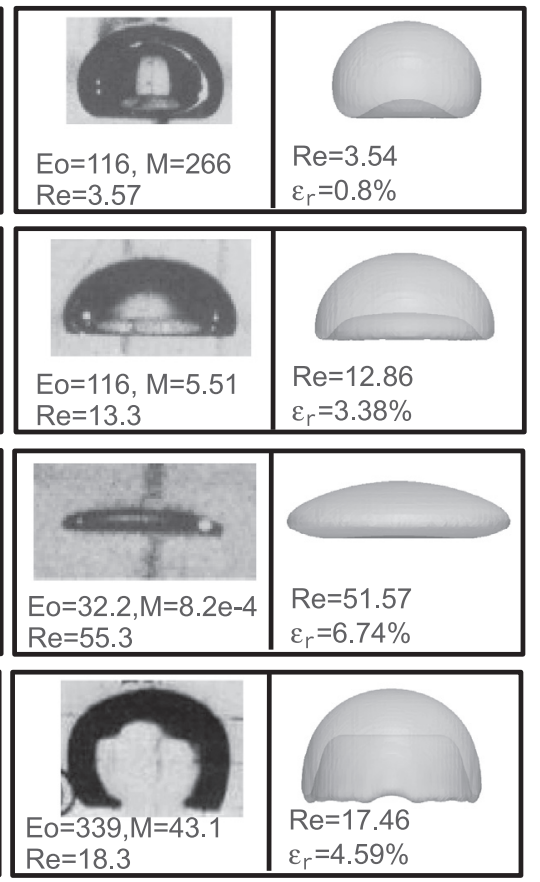

Experiment

CLS method

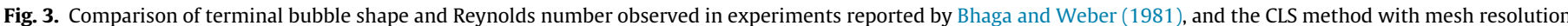
$h=d / 30$. The error in the Reynolds number is calculated by $\varepsilon_{r}=\left|R e_{\text {exp }}-R e_{\text {num }}\right| R e_{e x p}^{-1}$. All numerical experiments were carried out using $\eta_{\rho}=100, \eta_{\mu}=100$.

Table 4

Influence of the domain size $\left(D_{\Omega}\right)$ on the Reynolds number, $E o=116, M=41.1, \eta_{\rho}=$ 100 and $\eta_{\mu}=100$. Experimental reference $R e=7.16$ (Bhaga and Weber, 1981). Mesh configuration and domain are shown in Fig. 1.

\begin{tabular}{lllll}
\hline Mesh & Cell size & $\left(D_{\Omega}, H_{\Omega}\right)$ & Re & Relative error $\left(\varepsilon_{r}\right)(\%)$ \\
\hline$M_{4}$ & $h=d / 30$ & $(4 d, 12 d)$ & 6.44 & 10.01 \\
$M_{5}$ & $h=d / 30$ & $(6 d, 12 d)$ & 6.78 & 5.30 \\
$M_{3}$ & $h=d / 30$ & $(8 d, 12 d)$ & 6.94 & 3.03 \\
\hline
\end{tabular}

evolves to steady state for all Morton numbers, however, the Morton number has different effects on the time evolution of the bubble velocity. At large $M$ the Reynolds number quickly increases to a constant value, whereas at low $M$ the Reynolds number first increases to a maximum value and then decreases until steady state value. Moreover, as the Morton number increases the overshoot on $\mathrm{Re}$ is more pronounced, indicating that the bubble motion has a tendency to reduce their stability.

An important aspect to get accurate simulations is the mass conservation of the bubble phase. The mass gain or loss may affect the shape of the interface and also the dynamics of the problem. Fig. 5b shows the satisfaction of this requirement by illustrative simulations for $E o=116,1<M<10^{3}, \eta_{\rho}=100, \eta_{\mu}=100$ and $h=d / 30$. As the bubble is rising in the liquid, the instantaneous mass is calculated and compared with the initial mass. The mass error is then calculated by the expression $\Delta m=(m(t)-m(0)) /$

(a)
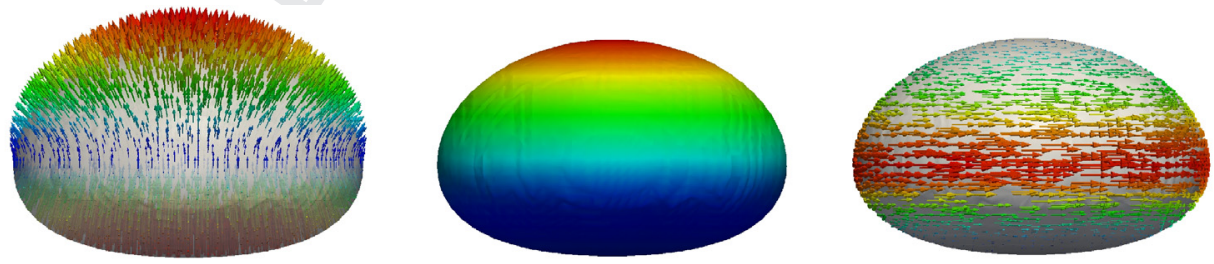

(b)
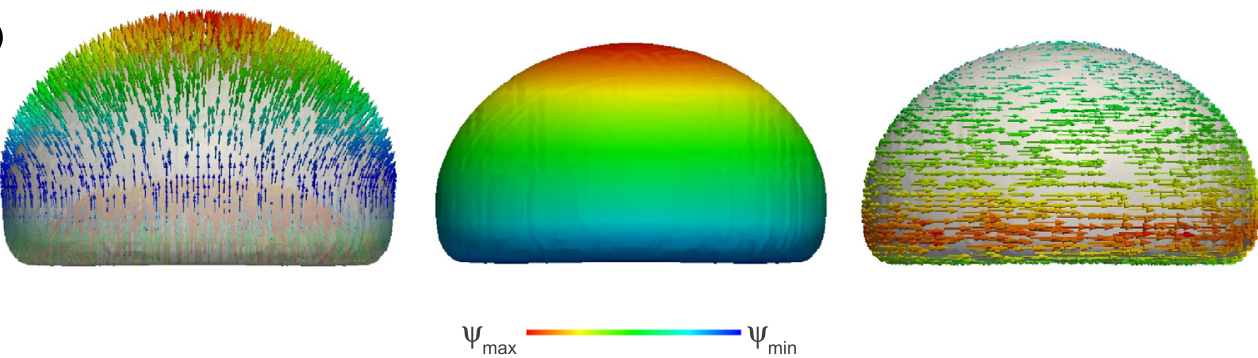

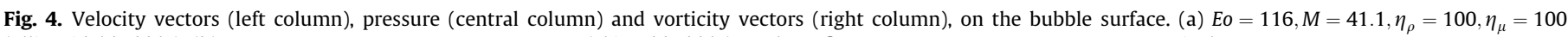
(ellipsoidal bubble). (b) $E o=116, M=41.1, \eta_{\rho}=100, \eta_{\mu}=100$ (skirted bubble). In these figures, $\psi \in\{\|\mathbf{v}\|, p, \Omega=\nabla \times \mathbf{v}\}$ respectively. 
Table 5

A comparison of our computational results against experimental results of Bhaga and Weber (1981) and numerical results reported by Hua et al. (2008) using the fronttracking method. Mesh configuration is shown in Fig. 1.

\begin{tabular}{|c|c|c|c|c|c|}
\hline \multirow[t]{2}{*}{ Eo } & \multirow[t]{2}{*}{$M$} & \multicolumn{3}{|l|}{$R e$} & \multirow[t]{2}{*}{ Mesh } \\
\hline & & $\begin{array}{l}\text { Bhaga and Weber } \\
\text { (1981) }\end{array}$ & $\begin{array}{l}\text { Hua et al. } \\
(2008)\end{array}$ & Present & \\
\hline 116 & 848 & 2.47 & 2.317 & 2.29 & $M_{3}$ \\
\hline 116 & 266 & 3.57 & 3.621 & 3.54 & $M_{3}$ \\
\hline 116 & 41.1 & 7.16 & 7.0 & 6.94 & $M_{3}$ \\
\hline 116 & 5.51 & 13.3 & 13.17 & 12.86 & $M_{3}$ \\
\hline 116 & 131 & 20.4 & 19.88 & 19.38 & $M_{3}$ \\
\hline 32.3 & $8.2 \times 10^{-4}$ & 55.3 & 52.96 & 51.57 & $M_{3}$ \\
\hline 243 & 266 & 7.77 & 8.397 & 7.68 & $M_{3}$ \\
\hline 339 & 43.1 & 18.3 & 17.91 & 17.46 & $M_{7}$ \\
\hline
\end{tabular}

$m(0)$ where $m(t)=\int \phi d V$. From Fig. $5 \mathrm{~b}$, it is demonstrated that the instantaneous mass of the bubble is conserved fairly well.

\subsubsection{Drag coefficient and comparison with empirical correlation}

Now, the accuracy of the CLS method is examined by comparison of the drag coefficient with experimental correlations and theoretical predictions. The drag coefficient $\left(C_{D}\right)$ can be obtained from a simulation where a bubble is released in an initially quiescent liquid. Thus, from a steady state balance in the vertical direction, $C_{D}$ can be computed from the terminal rise velocity $U_{T}$ :

$C_{D}=\frac{4\left(\rho_{l}-\rho_{g}\right)\|\mathbf{g}\| d}{3 \rho_{l} U_{T}^{2}}$

Bhaga and Weber (1981) proposed an experimental correlation between $C_{D}$ and $R e$ for fluids with Morton number $M>4 \times 10^{-3}$ :

$C_{D}=\left((2.67)^{0.9}+(16 / R e)^{0.9}\right)^{1 / 0.9} \quad M>4 \times 10^{-3}$

The numerical results are also compared against theoretical predictions of Joseph (2003), which was derived based on the theory of viscous potential flow. The drag coefficient is given as:

$C_{D}=0.445\left(6+\frac{32}{R e}\right)$

Hadamard (1911) and Rybczynski (1911) generalize the Stokes result for the viscous drag force on a solid sphere, to fluid particles of arbitrary and finite internal viscosity. From integration of pressure and shear stress on the fluid particle surface, they infer the drag coefficient at very low Re:

$C_{D}=\frac{8}{\operatorname{Re}} \frac{2+3 \eta_{\mu}^{-1}}{1+\eta_{\mu}^{-1}} \quad R e \ll 1$

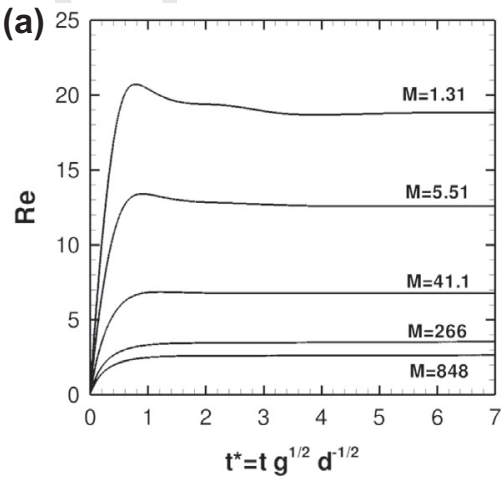

For the sake of comparison, a set of numerical simulations were carried out to calculate $C_{D}$ from Eq. (24). Fig. $6 \mathrm{~b}$ shows the terminal Reynolds number as a function of dimensionless time, with Eo $=10,10^{-3} \leqslant M \leqslant 10^{2}, \eta_{\rho}=100$ and $\eta_{\mu}=100$; whereas, Fig. 6a shows the drag coefficient as a function of the Reynolds number. As $M$ decreases, the bubble deformation increases and the spherical bubble approximation (see Eq. (27)) is valid for only high $M$, as one would indeed expect; moreover, numerical predictions of $C_{D}$ are in good agreement with Eqs. (25) and (26). In addition, the flow number $(F)$ and the velocity number $(V)$ defined as:

$F=g\left(\frac{d^{8} \rho_{l}^{5}}{\sigma \mu_{l}^{4}}\right)^{1 / 3}$

$V=U_{t}\left(\frac{d^{2} \rho_{l}^{2}}{\sigma \mu_{l}}\right)^{1 / 3}$

are evaluated from the numerical results given in Fig. 6 and Table 5 for $E o=10$ and $E o=116$ respectively. These results are compared to the following correlations reported by Rodrigue (2001):

$V=\frac{F}{12\left(1+0.049 F^{3 / 4}\right)}$

$V=\frac{F}{12(1+0.0185 F)^{3 / 4}}$

From Fig. 7 it is observed that numerical predictions are in good agreement with the correlations given by Eqs. (30) and (31).

\subsubsection{Effect of density ratio}

To further investigate the effect of density ratio on the terminal velocity $\left(U_{t}\right)$ and bubble shape, we simulate a bubble rising under buoyancy for $E o=39.4, M=0.065, \eta_{\mu}=6.37 \times 10^{3} \quad$ and $\eta_{\rho}=\{10,100,714\}$. Physical properties were chosen so that experimental data reported by Hnat and Buckmaster (1976) are equivalent to dimensionless parameters used in this section. Since the experiment was done in a large container with negligible wall effects, we use a very large computational domain with $\left(D_{\Omega}, H_{\Omega}\right)=(8 d, 16 d)$, which corresponds to the mesh $M_{6}$ in Fig. 1c. Fig. 8a shows the rise velocity and terminal bubble shapes as a function of the density ratio, whereas Fig. $8 \mathrm{~b}$ shows the time evolution of bubble sphericity. It can be seen that the bubble shapes are not affected by density ratio, as shown in Fig. 8a and b. The rise velocity is sensitive to $\eta_{\rho}$, indeed, a lower density ratio provides a lower value of terminal velocity $U_{T}$, such that the bubble will reach smaller distances in the same period of time. In addition, the sensitivity of rise velocity to density ratio is drastically reduced if $\eta_{\rho} \geqslant 100$ (see Fig. 8a). Table 6 shows the relative error on the

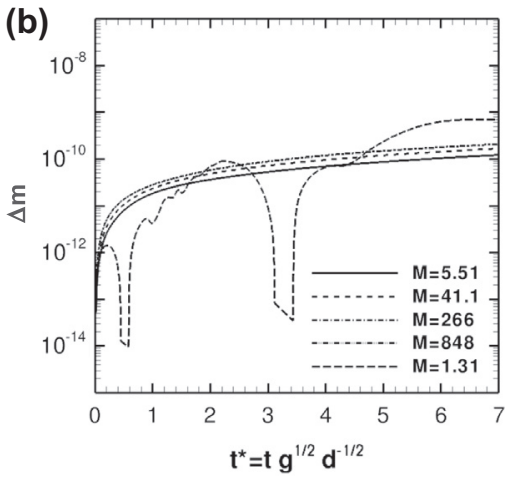

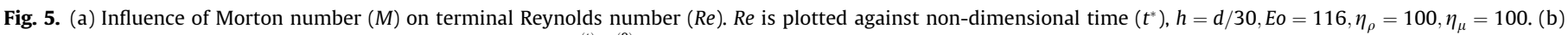
Mass conservation of bubble phase, $E o=116, M=266, \Delta m=\frac{m(t)-m(0)}{m(0)}$ where $m(t)=\int_{\Omega} \phi(\mathbf{x}, t) d V$. 

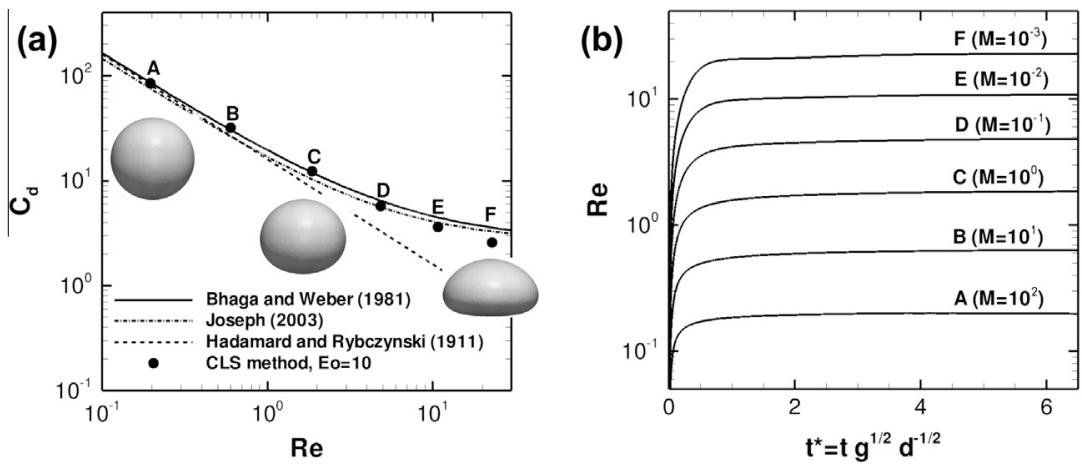

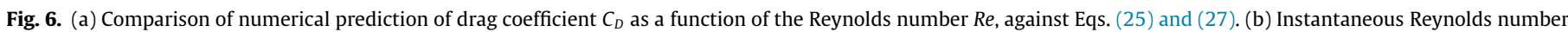
as a function of the dimensionless time $t^{*}$. Physical conditions are given by Eo $=10, \eta_{\rho}=100, \eta_{\mu}=100,10^{-3} \leqslant M \leqslant 10^{3}$ (low viscosity values).

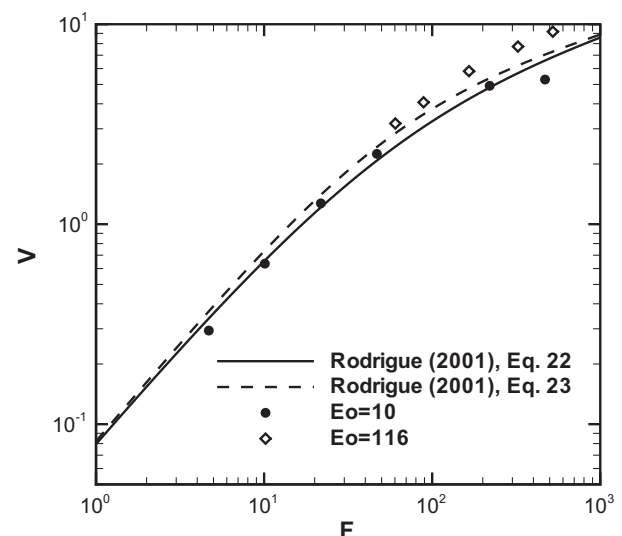

Fig. 7. Velocity number $(V)$ as a function of the flow number $(F)$. Correlations are taken from Rodrigue (2001).

terminal rise velocity. It can be observed that as the density ratio increases, the relative error is reduced (see Table 7).

\subsubsection{Effect of viscosity ratio}

The effect of viscosity ratio is evaluated by comparing the terminal velocity and shape for $E o=39.4, M=0.065$, $\eta_{\mu}=\left\{10,100,6.37 \times 10^{3}\right\}$ and $\eta_{\rho}=100$. As in the above test cases, the mesh $M_{6}$ is used to perform the present simulations (see Fig. 1c). From Fig. 9a, it is noted that as the value of viscosity ratio is increased, terminal velocity tends to collapse into a single curve. Effect of $\eta_{\mu}$ on terminal shape is presented in Fig. 9b. In addition, it is found that the effect of viscosity ratio on bubble shape is
Table 6

Effect of density ratio on terminal velocity. Here, $U_{T}^{*}=U_{T} d^{-1 / 2} g^{-1 / 2}$ and $\varepsilon_{r}=\left|U_{T}-U_{T, \text { exp }}\right| U_{T, \text { exp }}^{-1}$. The reference solution $U_{T, \text { exp }}=0.215 \mathrm{~m} / \mathrm{s} \quad\left(U_{T, \text { exp }}^{*}=0.6226\right)$ was obtained experimentally by Hnat and Buckmaster (1976) at Eo=39.4 $M=0.065, \eta_{\rho}=714$ and $\eta_{\mu}=6.67 \times 10^{3}$.

\begin{tabular}{rllllll}
\hline \multicolumn{1}{c}{$\eta_{\rho}$} & $\eta_{\mu}$ & Eo & $M$ & $U_{T}^{*}$ (CLS method) & $\varepsilon_{r}(\%)$ & Mesh \\
\hline 10 & $6.67 \times 10^{3}$ & 39.4 & 0.065 & 0.5833 & 6.31 & $M_{6}$ \\
100 & $6.67 \times 10^{3}$ & 39.4 & 0.065 & 0.6098 & 2.06 & $M_{6}$ \\
714 & $6.67 \times 10^{3}$ & 39.4 & 0.065 & 0.6118 & 1.73 & $M_{6}$
\end{tabular}

stronger than on bubble velocity, however, the variation of bubble rising velocity is less than $1 \%$ when the viscosity ratio changes from 100 to $6.67 \times 10^{3}$. The aforementioned results are in agreement with numerical findings reported by Hua et al. (2008).

\subsubsection{Wake patterns}

The influence of bubble shape on the flow pattern of the wake has been also investigated. In the experiment of Bhaga and Weber (1981) hydrogen bubble tracers were used to get the flow visualization, whereas shadow-graph and Schlieren techniques were employed by Hnat and Buckmaster (1976) to study the wake structure of spherical cap bubbles. Fig. 10a shows the terminal bubble wake for the case $E o=39.4, M=0.065, \eta_{\rho}=714$, $\eta_{\mu}=6670$. The closed toroidal wake and bubble shape predicted by the CLS method are in close agreement with the experimental findings of Hnat and Buckmaster (1976), furthermore, a comparison of the present results with numerical simulations reported by Ryskin and Leal (1984), Yue et al. (2007) and Gueyffier et al. (1999) are presented in Fig. 10a, which confirm the accuracy of the present simulations. Fig. $10 \mathrm{~b}$ and $\mathrm{c}$ shows results obtained in
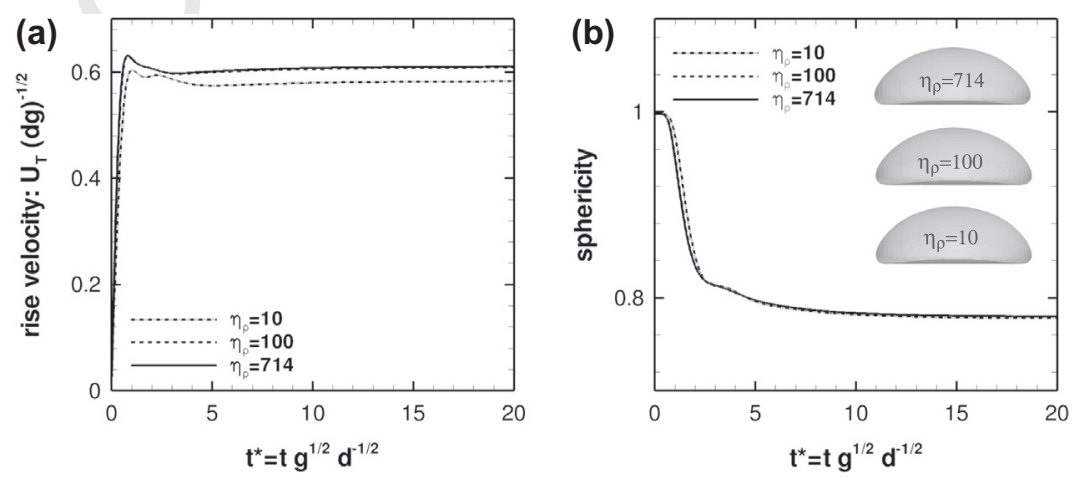

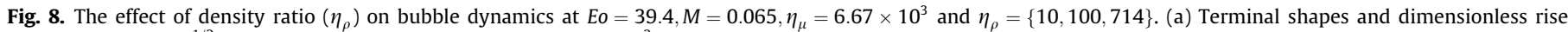
velocity $\left(\mathbf{v} \cdot \mathbf{e}_{y} g^{-1 / 2} d^{1 / 2}\right)$ versus dimensionless time. (b) Sphericity $\left(\pi d^{2} / \int_{\Omega}|\nabla \phi| d V\right)$ versus dimensionless time. 
Table 7

Effect of viscosity ratio on terminal velocity. Here, $U_{T}^{*}=U_{T} d^{-1 / 2} g^{-1 / 2}$ and $\varepsilon_{\mathrm{r}}=\left|U_{T}-U_{T, \text { exp }}\right| U_{T, \text { exp }}^{-1}$. The reference solution $U_{T, \text { exp }}=0.215 \mathrm{~m} / \mathrm{s} \quad\left(U_{T, \text { exp }}^{*}=0.6226\right)$ was obtained experimentally by Hnat and Buckmaster (1976) at Eo $=39.4$, $M=0.065, \eta_{\rho}=714$ and $\eta_{\mu}=6.67 \times 10^{3}$.

\begin{tabular}{lllllll}
\hline$\eta_{\rho}$ & $\eta_{\mu}$ & Eo & $M$ & $U_{T}^{*}$ (CLS method) & $\varepsilon_{r}(\%)$ & Mesh \\
\hline 100 & 10 & 39.4 & 0.065 & 0.5699 & 8.46 & $M_{6}$ \\
100 & 100 & 39.4 & 0.065 & 0.6038 & 3.02 & $M_{6}$ \\
100 & $6.67 \times 10^{3}$ & 39.4 & 0.065 & 0.6118 & 2.06 & $M_{6}$ \\
\hline
\end{tabular}

the present work and also numerical and experimental wakes reported on Hua et al. (2008) and Bhaga and Weber (1981) respectively. These cases correspond to dimensionless parameters $E o=116, M=0.065, \eta_{\rho}=100, \eta_{\mu}=100 \quad$ (Fig. 10b), and $E o=292, M=26.7, \eta_{\rho}=100, \eta_{\mu}=100 \quad$ (Fig. $10 \mathrm{~b}$ and c). Secondary wake recirculations were observed for the aforementioned cases (see Fig. 10b and c), which could explain the bright zones (caused by refraction effects (Bhaga and Weber, 1981)) just behind the bubble rim at the experimental images reported by Bhaga and Weber (1981); moreover, the present results performed by the CLS method are confirmed by numerical predictions reported by Hua et al. (2008) using the front-tracking method.

\subsubsection{Effect of convective schemes}

Further simulations have been performed in order to study the influence of the convective scheme used to discretize Eq. (6) on the rising velocity and bubble shapes. Fig. 11 shows numerical results for $E o=39.4, M=0.065, \eta_{\rho}=714, \eta_{\mu}=6.67 \times 10^{3}$ and using the mesh $M_{6}$ (see Fig. 1c) which corresponds to $h=d / 30$. The reader is referred to Balcázar et al. (2014) for technical details on the application of flux limiters to discretize the convective term of momentum equation on unstructured grids. Following the work of Balcázar et al. (2014), the finite-volume discretization of the convective term on momentum equation is based on the use of flux limiters, $L(\theta)$, given by

$$
L(\theta) \equiv \begin{cases}1 & \text { Central difference limiter }(C D) \\ \max \{0, \min \{2 \theta, 1\}, \min \{2, \theta\}\} & \text { TVD Superbee limiter } \\ \frac{\theta+|\theta|}{1+|\theta|} & \text { TVD Van-Leer limiter } \\ 0 & \text { First-order upwind limiter }\end{cases}
$$

where $\theta$ is a monitor variable defined as the upwind ratio of consecutive gradients of the velocity components. Regarding the numerical results, Fig. 11a illustrates the effect of flux limiters on the rising velocity. A close-up on the time $t \approx 0.8$ shows that the upwind limiter underestimates the overshoot on the rising velocity, while the other flux limiters lead to similar results. Fig. 11b and c shows that sphericity and shape of the bubbles are in close agreement, independently of the flux limiter used, however it is noted a slight deviation when an upwind limiter is used in comparison with the other ones (see Table 8). Regarding the rising velocity, the deviation of numerical results from experimental results, $\varepsilon_{r}$, was found to be slightly minor with the $C D$ limiter, as shown in Table 8.

Thus, the calculated drag coefficients, bubble shapes, Reynolds number and wake structure are consistent with the existing correlations and experimental data reported in the literature. These various comparisons serve to validate the accuracy and versatility of the CLS method.

\subsection{Hydrodynamic interaction of bubble pairs}

In this section, the CLS method is used to explore the complex interaction of two spherical and ellipsoidal bubbles for a wide range of flow conditions and different geometrical configurations. Fig. 12 shows the computational system and mesh distribution. Current simulations are performed in a rectangular domain with periodic boundary conditions in the vertical direction, $y$, and Neumann boundary conditions at lateral boundaries. The distance between the bubble centroids and the lateral walls was set to be larger than 4 bubble diameters in order to minimize the confinement effect (see Table 4). From Fig. 12b the domain is divided in $2.9 \times 10^{6}$ hexahedral volumes, which is equivalent to 30 control volumes per bubble diameter $(c v / d)$. Numerical experiments were carried out under conditions of different $E o$ and $M$ numbers. In addition, the interaction between a pair of bubbles is influenced by their center-to-center separation distance, $s$, and their configuration angle, $\theta$, as shown in Fig. 12a. As initial condition, the bubbles are at rest and a spherical shape is assumed. Regarding the discretization of the convective term of momentum Eq. (6), a CD scheme is used for cases with low Reynolds number, which include interactions of spherical bubbles $\left(E 0=0.36, M \leqslant 5 \times 10^{-8}\right)$. Moreover, a TVD scheme (Van-Leer flux limiter) is used in order to avoid numerical instabilities at cases with high Reynolds number, which include interactions of ellipsoidal bubbles $\left(E o=4, M=1 \times 10^{-9}\right)$ and spherical bubbles with $M=1 \times 10^{-8}$.

\subsubsection{Spherical bubbles}

According to empirical findings of Takemura and Magnaudet (2003) and Sanada et al. (2009), and numerical studies of Legendre and Magnaudet (2003), when two spherical bubbles rise side by side, a horizontal force is induced and it causes either repulsion or attraction between the bubbles, as result of the competition between vortical and irrotational interaction mechanisms.
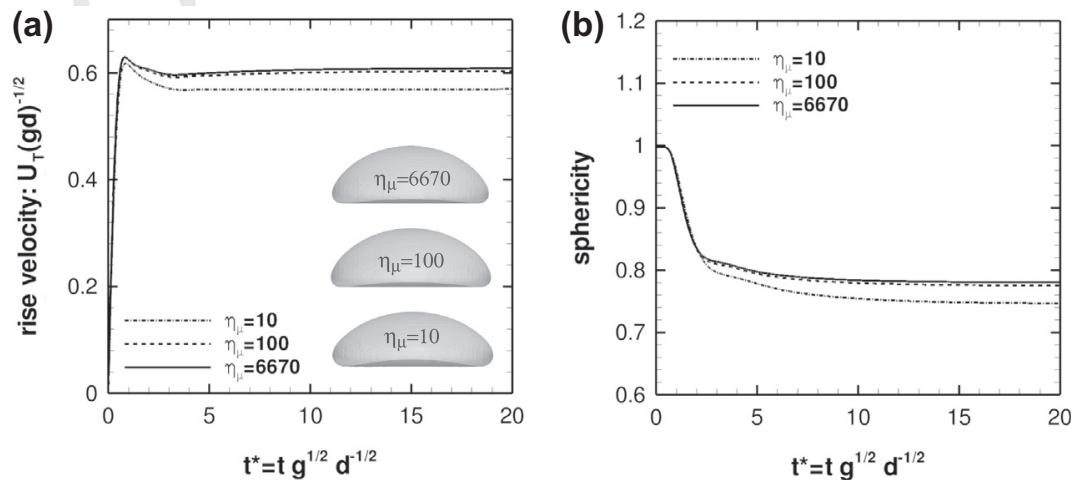

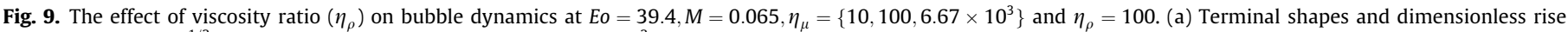
velocity ( $\left.\mathbf{v} \cdot \mathbf{e}_{y} g^{-1 / 2} d^{1 / 2}\right)$ versus dimensionless time. (b) Sphericity $\left(\pi d^{2} / \int_{\Omega}|\nabla \phi| d V\right)$ versus dimensionless time. 
(a) $\mathrm{Eo}=39.4$ $M=0.065$

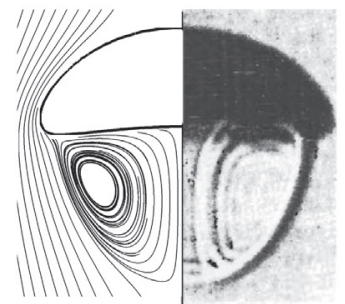

Present work Hnat and Buckmaster (1976)

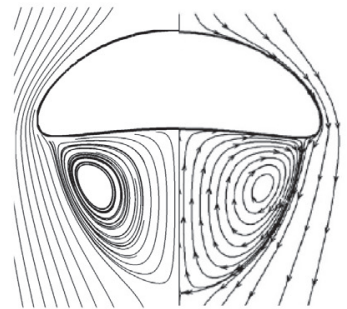

Present work Yue et al. (2007)

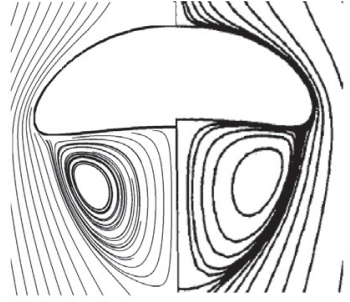

Present work Ryskin and Leal (1984)

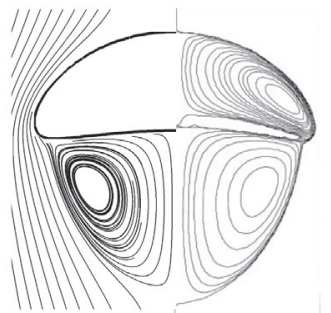

Present work Gueyffier et al. (1999)

(b) $E o=116$

$M=0.962$

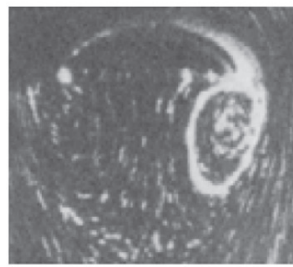

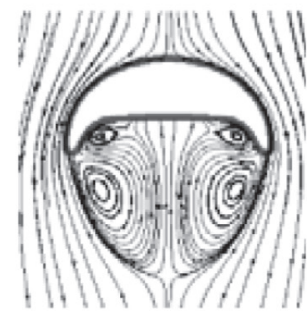

Bhaga and Webber (1981) Hua and Lou (2007)

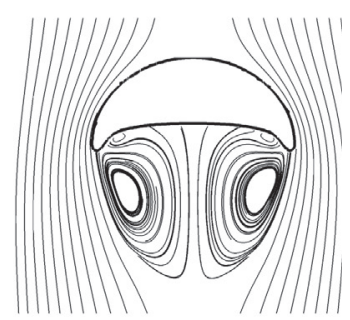

Present work (c) Eo $=292$

$M=26.7$

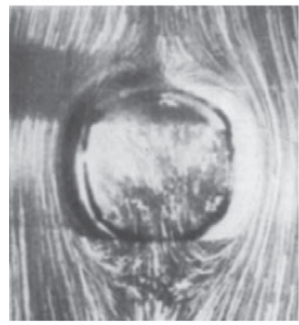

Bhaga and Webber (1981)

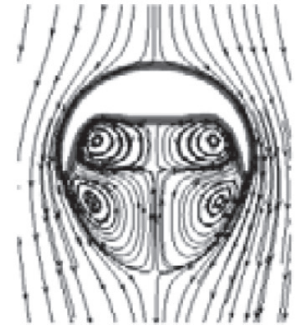

Hua and Lou (2007)

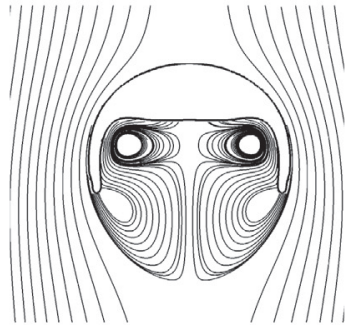

Present work

Fig. 10. Comparisons of experimental and numerical results previously published against present results (CLS method) for the wake pattern. (a) $E o=39.4, M=0.065, \eta_{\rho}=714, \eta_{\mu}=6.67 \times 10^{3}$. Reference results are reported in Hnat and Buckmaster (1976), Ryskin and Leal (1984), Yue et al. (2007) and Gueyffier et al. (1999). (b) $E o=116, M=0.962, \eta_{\rho}=100, \eta_{\mu}=100$. Reference results are reported in Bhaga and Weber (1981) and Hua et al. (2008). (c) $E o=292, M=26.7, \eta_{\rho}=100, \eta_{\mu}=100$. Reference results are reported in Bhaga and Weber (1981) and Hua et al. (2008). The meshes used in present simulations are $M_{6}$ (a), $M_{3}$ (b) and $M_{7}$ (c). See Fig. 1c for mesh description.

Thus, the lateral force changes sign for a certain critical Reynolds number $\left(R e_{c}\right)$. For instance, Legendre and Magnaudet (2003) numerically studied the motion of a pair of spherical rising bubbles aligned horizontally by using DNS and predicted that $\operatorname{Re}_{c} \approx 30$ if the horizontal distance between the bubbles is $s=1.5 \mathrm{~d}$ and $\theta=0$, a value confirmed by the experimental results of Takemura and Magnaudet (2003).

Following previous results, a set of numerical experiments were performed for $E o=0.34,4.8 \times 10^{-6} \leqslant M \leqslant 1 \times 10^{-8}, \quad \eta_{\rho}=100$, $\eta_{\mu}=100, s=1.5 d$ and $\theta=0$. From Fig. 13a, in simulations where $\operatorname{Re} \lesssim 32$ the bubbles separate from each other. On the other hand, for cases with $R e \gtrsim 32$ the bubbles approach each other (see Fig. 16). Such behavior has been explained by Legendre and Magnaudet (2003) in terms of the vorticity: when Re is large enough for the vorticity to remain confined in a boundary layer whose thickness is small compared to the distance between the two bubbles, the interaction is dominated by the irrotational mechanism, consequently, the transverse force is then attractive. However, when viscous effects are dominant, the vorticity spreads out about each bubble until it is canceled in the gap by the vorticity existing around the other bubble, resulting in a repulsive transverse force for small values of $R e$. The numerical prediction of the transitional Reynolds number $\left(R e_{c} \approx 32\right)$ given by the CLS method, is in excellent agreement with numerical and experimental results previously published (Legendre and Magnaudet, 2003; Yu et al., 2011; Bonometti and Magnaudet, 2007; Sanada et al., 2009; Takemura and Magnaudet, 2003).

The effect of the initial configuration angle on the hydrodynamic interaction between two spherical bubbles is studied for $s=1.5 d, 0^{\circ} \leqslant \theta \leqslant 90^{\circ}, E o=0.34, M=1 \times 10^{-8}$ (or $\operatorname{Re}=61.1$ for 

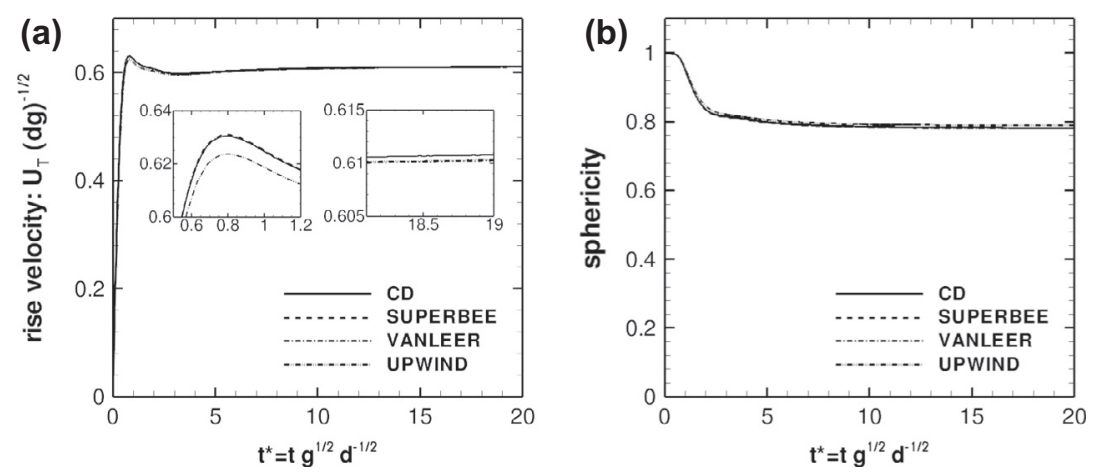

(c)
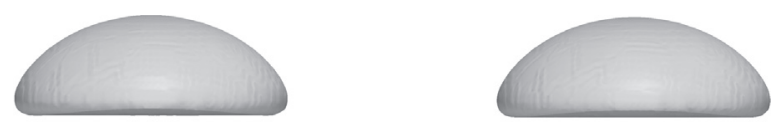

CD

Superbee

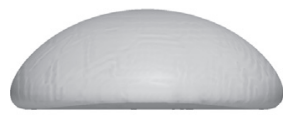

Van-Leer

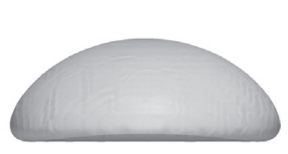

Upwind

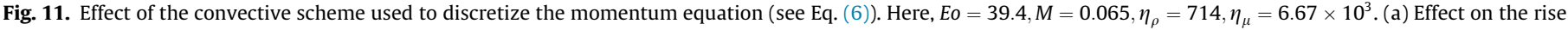
velocity. (b) Effect on the sphericity (bubble shape). (c) Qualitative comparison of terminal bubble shape.

Table 8

Effect of flux limiters used to discretize the momentum equation on terminal rising velocity. Here, $U_{T}$ is the terminal rise velocity $\left(\mathbf{v}_{c} \cdot \mathbf{e}_{y}\right.$ at $\left.t^{*}=20\right)$ and $\varepsilon_{r}=\left|U_{T}-U_{T, \exp }\right| U_{T, \exp }^{-1}$ is the error in the computed velocity. The reference solution $U_{T, \exp }=0.215 \mathrm{~m} / \mathrm{s}\left(U_{T, \exp }(g d)^{-1 / 2}=0.6226\right)$ was obtained experimentally by Hnat and Buckmaster (1976) for $E o=39.4, M=0.065, \eta_{\rho}=714$ and $\eta_{\mu}=6.67 \times 10^{3}$.

\begin{tabular}{lllll}
\hline Flux limiter & $U_{T}(g d)^{-1 / 2}$ & $\varepsilon_{r}(\%)$ & Sphericity & Mesh \\
\hline CD & 0.6109 & 1.88 & 0.7818 & $M_{6}$ \\
Superbee & 0.6105 & 1.94 & 0.7802 & $M_{6}$ \\
Van-Leer & 0.6104 & 1.96 & 0.7805 & $M_{6}$ \\
Upwind & 0.6105 & 1.94 & 0.7890 & $M_{6}$ \\
\hline
\end{tabular}

the corresponding single bubble). The evolution of $s^{*}=2 s / d$ and $\theta$ are reported in Fig. 14 versus dimensionless time $t^{*}$ for different initial orientation angles $\theta_{0}$. Fig. 14b shows a decrease of the configuration angle as it evolves in time, which indicates a torque action on the bubbles that tends to align them side-by-side. The plot of the dimensionless distance (see Fig. 14a) indicates that the two bubbles being attracted for $0^{\circ} \leqslant \theta \leqslant \theta_{c}$ but repelled for $\theta_{c} \leqslant \theta \leqslant 90^{\circ}$, where $\theta_{c}$ is the angle of transition. From Fig. 14a, $\theta_{c}$ is between $30^{\circ}$ and $45^{\circ}$, which is in good agreement with the value $\theta_{c} \approx 37^{\circ}$ reported by Hallez and Legendre (2011). Regarding topology changes of interfaces for cases with attractive forces between the bubbles, these are consistent with numerical simulations reported by Yu et al. (2011) and Bonometti and Magnaudet (2007).

\subsubsection{Ellipsoidal bubbles}

In this section we study the interaction of two bubbles on ellipsoidal regime with $E o=4, M=1 \times 10^{-9}, \quad \eta_{\rho}=100, \eta_{\mu}=100$, $s=1.5 d, 0^{\circ}<\left.\theta\right|_{t=0}<90^{\circ}$. Fig. 17 shows the variation of shape and position for different initial configuration angles. For $\left.\theta\right|_{t=0}=0^{\circ}$, (a)

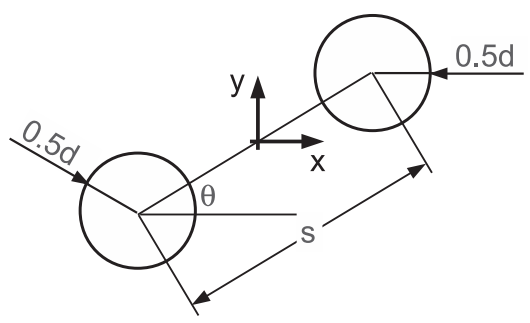

(b)

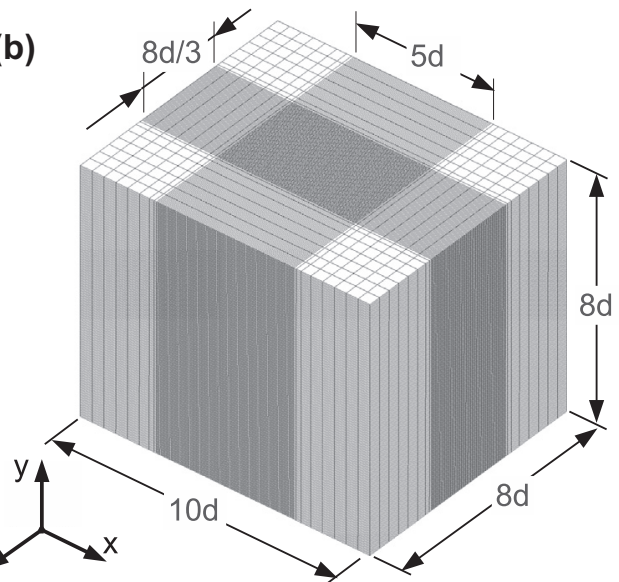

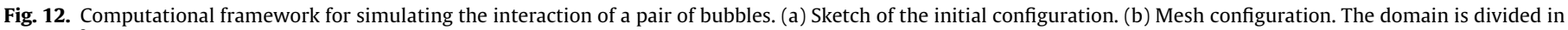
$2.9 \times 10^{6}$ hexahedral volumes, which is equivalent to $30 \mathrm{cv} / \mathrm{d}$. 

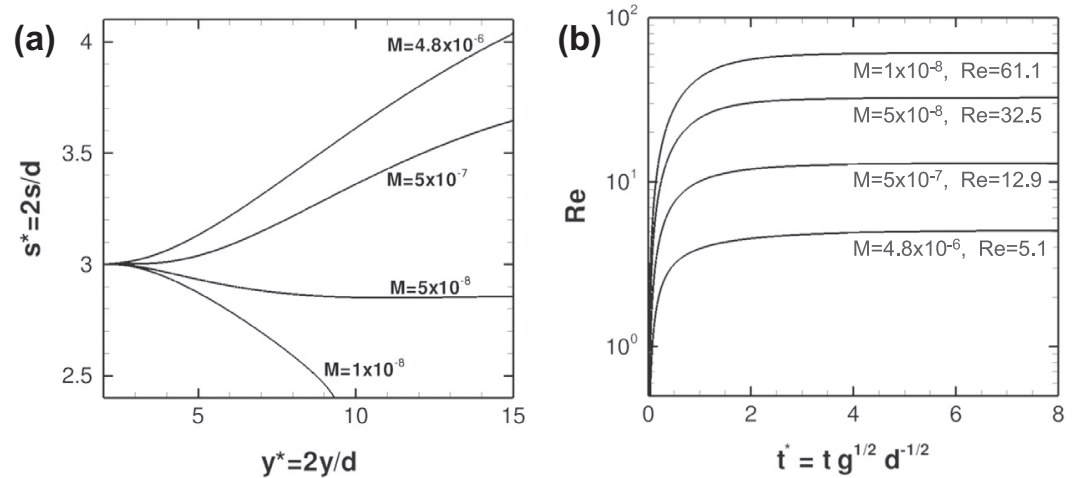

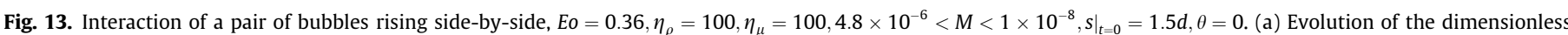

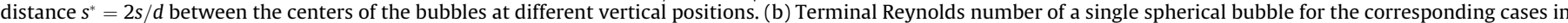
(a).
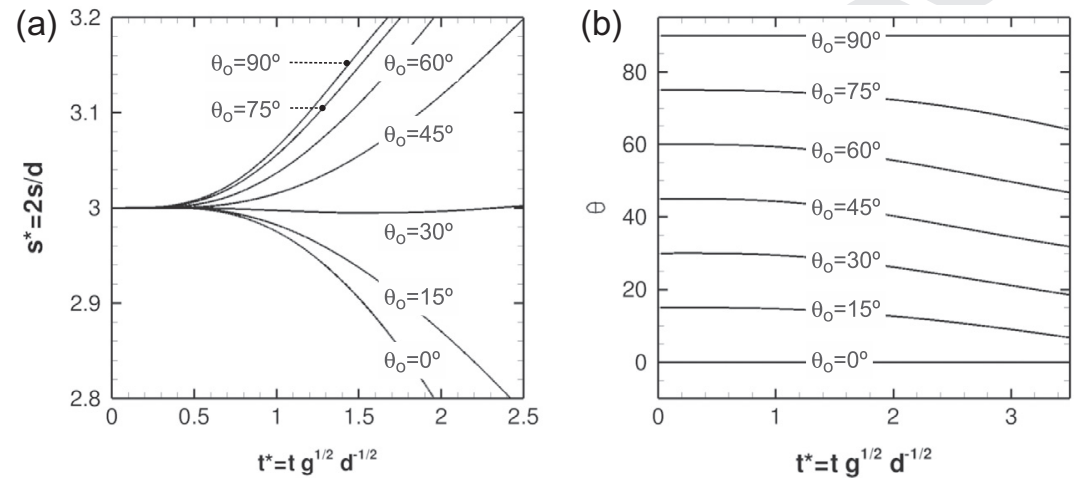

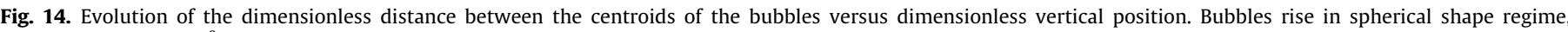
Eo $=0.34, M=1 \times 10^{-8}, \eta_{\rho}=100, \eta_{\mu}=100$.

the trajectory and shape of bubbles are symmetric about $y-z$ plane. For cases with $\left.\theta\right|_{t=0}=0^{\circ}$ and $\left.\theta\right|_{t=0}=15^{\circ}$, the bubbles separate each other until $t^{*} \approx 0.8$, where $s$ starts to decrease up to $t^{*} \approx 1$.7. From this time a repulsion force acts between the bubbles and $s$ continues to increase faster, as shown in Fig. $15 \mathrm{a}$. For $\left.\theta\right|_{t=0} \gtrsim 30^{\circ}$, the dynamic evolution of $s$ is the opposite than previous cases (see Fig. 15a), and attractive forces were observed, which lead to coalescence of small bubbles to form a larger one (see Fig. 17c).

Fig. 15b shows the time evolution of $\theta$ for different initial orientation angles. For $\left.\theta\right|_{t=0} \geq 30^{\circ}$ bubbles tend to align with vertical axis before to start the coalescence process. Indeed, the impact angle increases as $\left.\theta\right|_{t=0}$ is larger. For $\left.\theta\right|_{t=0}=15^{\circ}$, the orientation angle tends to align with the side-by-side configuration (see Fig. 15b). From previous findings, it is argued that deformation of bubbles has a strong effect on its interaction. In fact, the vorticity is larger for ellipsoidal bubbles than for spherical bubbles, since the vorticity generated at the interface is proportional to the curvature (Batchelor, 1967; Bunner and Tryggvason, 2003).

Fig. 18 shows the coalescence of two ellipsoidal bubbles rising in oblique alignment $\left(\theta=30^{\circ}\right)$. The pressure on the bubble surface
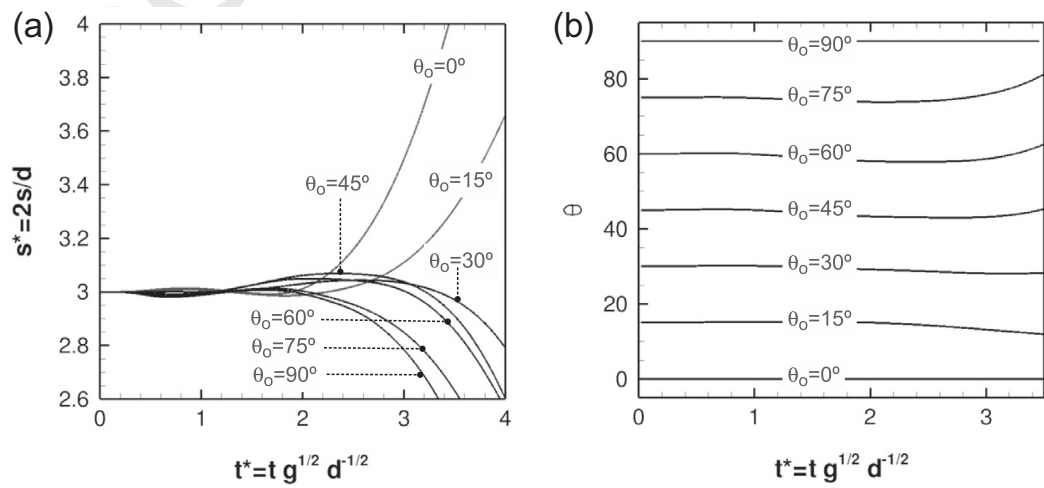

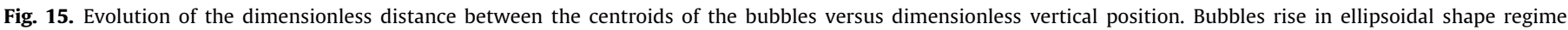
EO $=4.0, M=1 \times 10^{-9}, \eta_{\rho}=100, \eta_{\mu}=100$. 


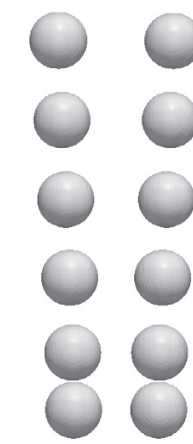

$\theta=0^{\circ}$
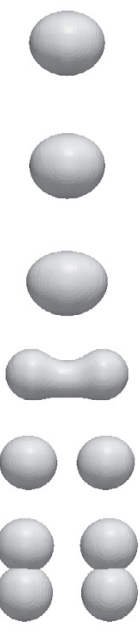

$\theta=0^{\circ}$

$\mathrm{Eo}=0.34, \mathrm{Re}=61.1$

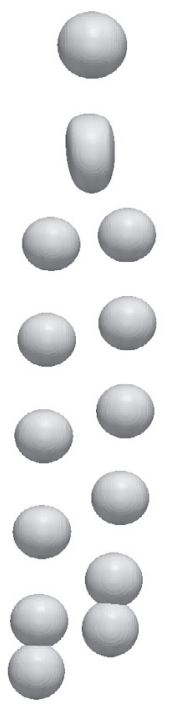

$\theta=30^{\circ}$

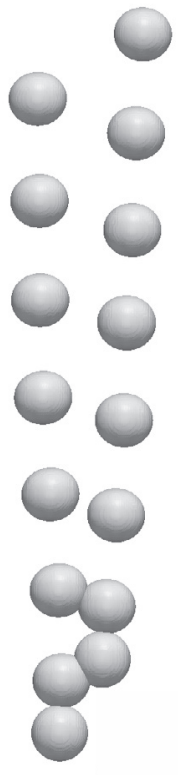

$\theta=75^{\circ}$

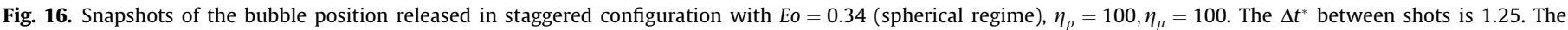
indicated Re number is defined based on the rise velocity of an isolated bubble under the same conditions.

and velocity vectors on the plane $x-y$ at $z=0$ of the domain are also presented for different times $t^{*}=\{4.4,5.0,6.0\}$. The shape of the larger bubble is different compared with spherical regime, thereby indicating that the coalescence dynamics is a function of the Eötvös number. In this regime, the wake behind the upper bubble creates an artificial lift force for the downstream bubble, which gradually moves in the wake of the leading bubble, moreover, a horizontal component of velocity is generated, which produces an alignment effect of bubbles (see Fig. 18, $t^{*}=4.4$ ). When the two bubbles are close to collide, a considerable asymmetrical deformation was observed, followed by the formation of a neck or meniscus between the bubbles (see Fig. $18, t^{*}=5.0$ ). Finally, two bubbles coalesce to form a big bubble at $t^{*}=6.0$ (see Fig. 18), which continues rising in wobbling regime. These

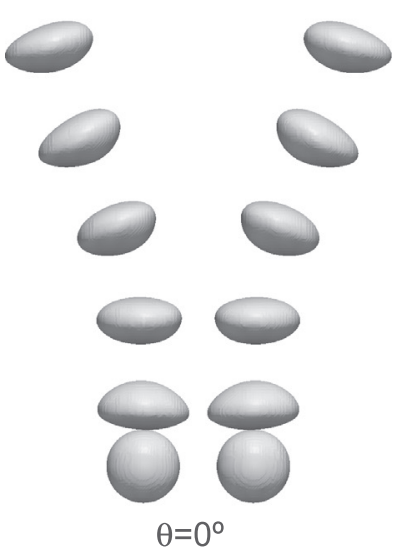

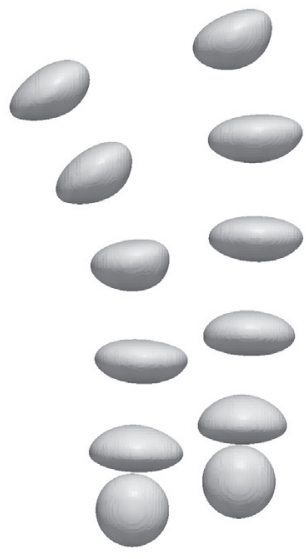

$\theta=15^{\circ}$

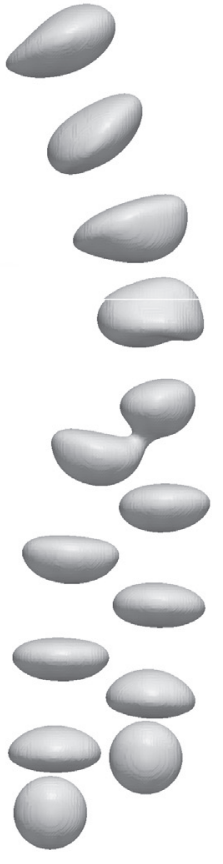

$\theta=30^{\circ}$

Fig. 17. Snapshots of the bubble position released in a staggered configuration with $E o=4$ (ellipsoidal regime), $M=1 \times 10^{-9}, \eta_{\rho}=100, \eta_{\mu}=100$. The $\Delta t^{*}$ between shots is 1.25. According to the Grace diagram (Clift et al., 1978), the Reynolds number corresponds to $R e \approx 700$, based on the rise velocity of an isolated bubble under the conditions mentioned above. 


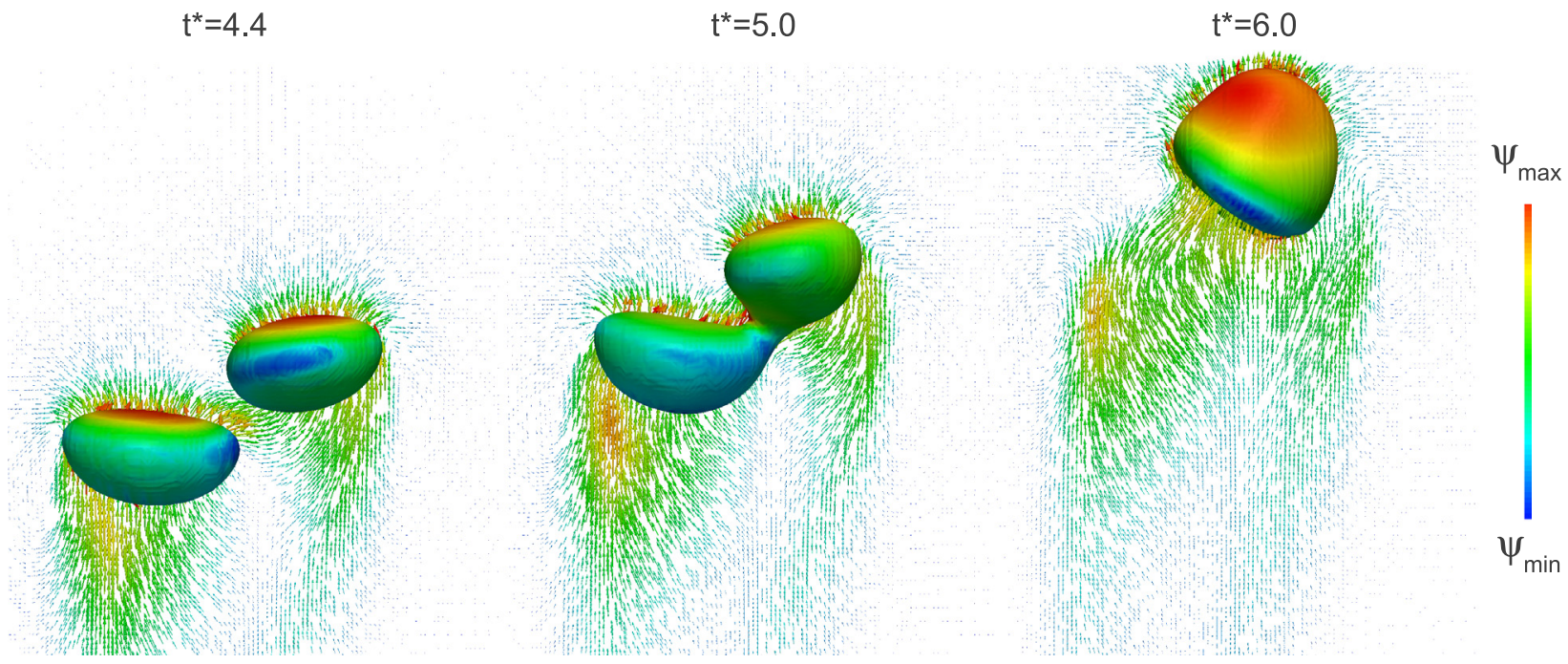

Fig. 18. Velocity field on the plane $x-y$ at $z=0$, and pressure field on the bubble surface for $E o=4, M=1 \times 10^{-9}, \eta_{\rho}=100, \eta_{\mu}=100, \theta=30^{\circ}$. Here $\psi \in\{p,\|\mathbf{v}\|\}$.

(a) (b)

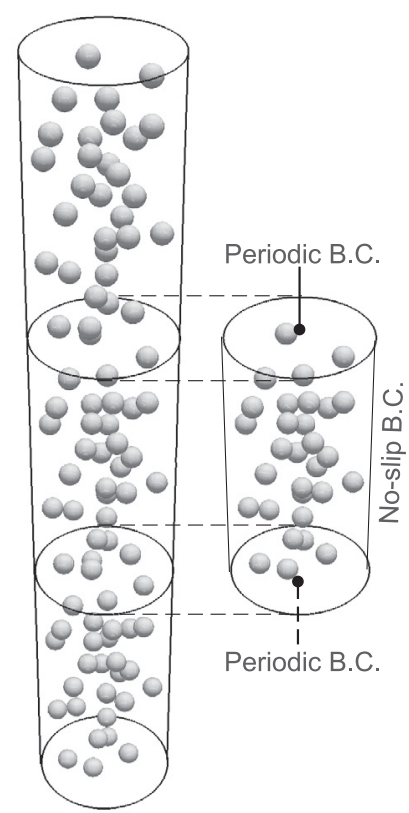

Fig. 19. Computational setup for simulation of multiple bubbles rising in a periodic vertical duct. (a) Initial condition (free bubble array) and Boundary conditions. (b) Mesh configuration (hexahedral cells).

topology changes are consistent with numerical simulations reported by Yu et al. (2011) using an adaptive Lattice-Boltzmann method.

\subsection{Multiple bubble interaction}

The bubbly flow is not a fully developed flow regime because given enough time, the bubbles may collide each other, and their agglomeration could lead to form large bubbles or the so-called slug flow. Moreover, in most industrial applications, the bubble flow rate is high and the flow is often referred to as churn flow, where the coalescence and break-up is strong. In general, the hydrodynamic of bubbly flow is still far to be understood, indeed, one major problem is the lack of proper experimental techniques to probe the bubbles (Mudde, 2005). However, for some systems, DNS can help us to understand the complex bubble interactions on bubbly flows. For instance, Tryggvason et al. (2013) have employed a front-tracking/finite-volume method to conduct DNS of bubbly flows with nearly spherical and ellipsoidal bubbles rising in a vertical channel with upward and downward flows and characterized the void fraction distribution and the velocity profile. Bunner and Tryggvason (2002), Bunner and Tryggvason (2003) and Esmaeeli and Tryggvason (2005) characterized the rise velocity, microstructure and velocity fluctuations on swarms of spherical and ellipsoidal bubbles using the front-tracking method. Smolianski et al. (2008) carried out two-dimensional simulations of the dynamics of a small group of bubbles using the level-set method. Liu and Valocchi (2012) and Cheng et al. (2010) have employed the Lattice-Boltzmann method to investigate the interactions of multiple bubbles rising in a vertical channel. In experimental studies, the coalescence can be prevented by the addition of salt to the liquid (Martínez-Mercado et al., 2007; Zenit et al., 2001), moreover, some numerical methods such as Front-Tracking introduced by Unverdi and Tryggvason (1992) and Tryggvason et al. (2001) have the ability to avoid the merging of interfaces by using a separate mesh for each bubble. In present simulations there is no such restriction and the bubble coalescence will be permitted.

Fig. 19 shows the computational domain, which is defined as a vertical cylinder bounded by a rigid wall, with gravity acting in the $-y$-direction. The size of the domain is $\left(D_{\Omega} \times H_{\Omega}\right)=(6.6 d \times 13.3 d)$, where $d$ is the initial bubble diameter, $D_{\Omega}$ is the cylinder diameter and $H_{\Omega}$ is the cylinder height. Imposed boundary conditions are non-slip at the rigid wall and periodic on the streamwise ( $y$-direction), as shown in Fig. 19a. In this way bubbles go out of the domain on the top side, and they come back in the domain again from the opposite side. The mesh was generated by a constant step extrusion of a two dimensional unstructured grid along the $y$-direction, which leads to a mesh conformed by $13 \times 10^{6}$ hexahedral volumes, as is illustrated in Fig. 19b. Therefore, the equivalent bubble diameter, $d$, is resolved by 30 mesh cells. An array of thirty spherical bubbles is initially placed in the periodic column following a random pattern, with $1.5 d$ defined as the minimum separation distance between two bubbles or bubble-wall (see Fig. 19a). This corresponds to a dilute bubbly flow, with an overall volumetric fraction of $\alpha=3.45 \%$. Non-dimensional parameters are fixed to $E o=2.5, M=5.9 \times 10^{-4}, \eta_{\rho}=10, \eta_{\mu}=10$, which corresponds to nearly spherical bubbles (Clift et al., 1978). 
(a)

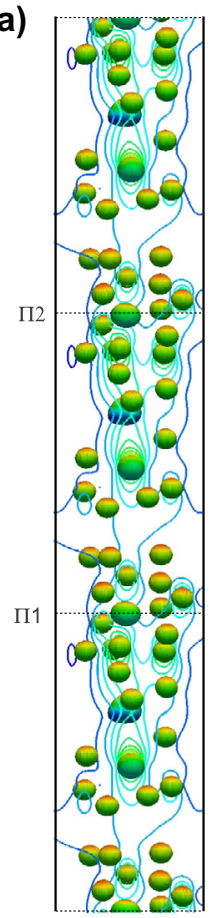

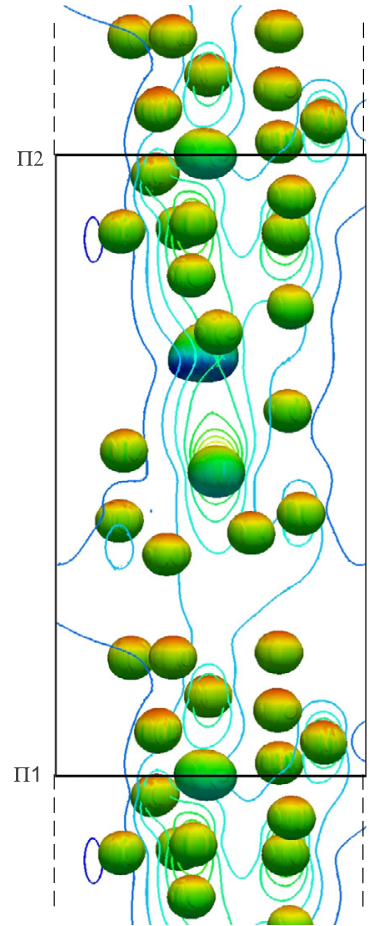

(b)

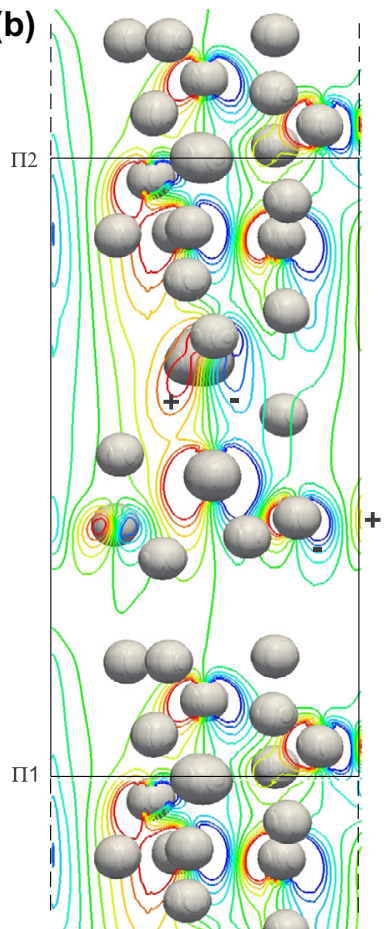

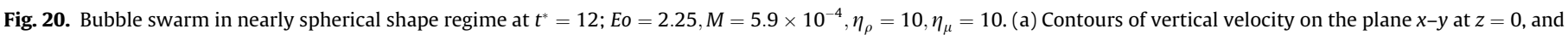
pressure distribution on the bubble surface. (b) Vorticity $\Omega \cdot \mathbf{e}_{z}$ on the plane $x-y$ at $z=0$.

(a)

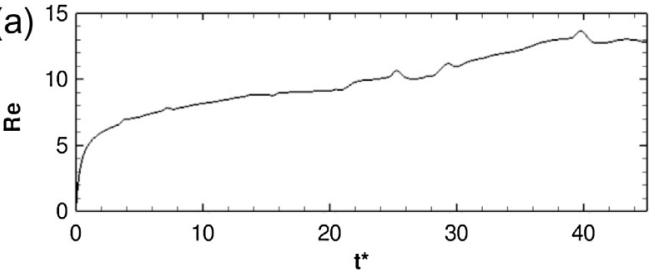

(b)

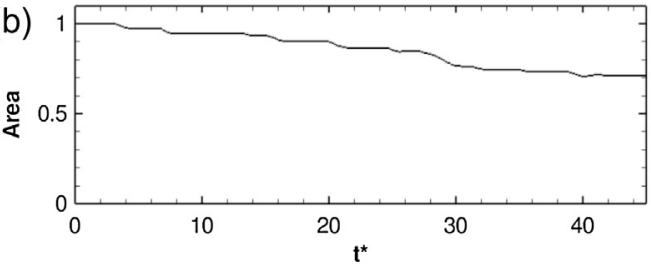

(c)

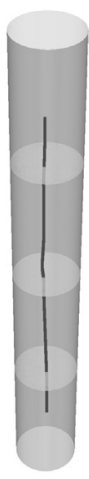

Fig. 20 shows an instantaneous snapshot of bubbly flow at the dimensionless time $t^{*}=12$, with $t^{*}=t(\mathrm{~g} / \mathrm{d})^{1 / 2}$. Fig. 20a illustrates the periodic behavior of the bubbles, where contours of vertical velocity $\left(\mathbf{v} \cdot \mathbf{e}_{y}\right)$ are plotted on the plane $x-y$ at $z=0$, and pressure distribution is represented on the bubble surface. Fig. 20b shows the vorticity contours, $\Omega \cdot \mathbf{e}_{z}$, on the plane $x-y$ at $z=0$, generated by the bubbles and the wall. The simulation was performed from $t^{*}=0$ to $t^{*}=46$, and it was stopped before the coalescence of all bubbles. Fig. 21a and b illustrates the time evolution of the average vertical velocity of the bubbles and its interfacial area respectively, indeed, a steady state behavior has not been achieved for the case reported in this section. In order to approximate the maximum flow length scale, the trajectory of the bubble swarm centroid $\mathbf{r}(t)$, has been calculated as follows:

$$
\mathbf{r}_{c}(t)=\mathbf{r}_{c}(0)+\int_{0}^{t} \mathbf{v}_{c}(t) d t
$$

with $\mathbf{v}_{c}(t)$ defined as the averaged velocity of the bubbles in the cylindrical domain $\Omega$,

$\mathbf{v}_{c}(t)=\frac{1}{V_{\Omega}} \int_{\Omega} \mathbf{v}(\mathbf{x}, t) \phi d V$

Therefore, the maximum length scale in vertical direction (y-axis) corresponds to 4 periodic domains, as is illustrated in Fig. 21c.

Fig. 22 shows the evolution of bubbly flow, including velocity field on the plane $x-y$ at $z=0$ and bubble distribution, for different times $t^{*}=\{12,24,38,46\}$. At the early stage, bubbles move upward through a basically stagnant liquid, and the rise trajectory of the bubbles hardly deviates from a straight line. Bubbles rise due to the buoyancy force and each bubble forms its own wake, which affects the motion of the nearby bubbles. As the time advances, trailing bubbles are attracted by the wake of the leading bubbles and coalescence processes are observed. This in turn, influences the bubble size distribution, interfacial area and bubble induced liquid flow, as shown in Fig. 22. The strong wake interaction promotes bubble agglomeration and the collision between them increases the coalescence of small bubbles to form larger ones. Moreover, Fig. 22 shows a major bubble concentration around the symmetry axis of the channel, whereas the bubbles do not collide with the wall. Hydrodynamics and gravity start to interact on global scales, and specific patterns are identified in the liquid circulation: the liquid flows up in the center of the circular duct and down close to the wall, as shown in Fig. 22. In present simulations, the cluster of bubbles form a chimney, which can be clearly seen in Fig. 22. This type of cluster formation is known to be the basic foundation for the transition from bubbly to slug flow. The trend for the bubbles to move to the center of the domain is consistent with simulation results reported by Cheng et al. (2010) and Yu et al. (2011). 


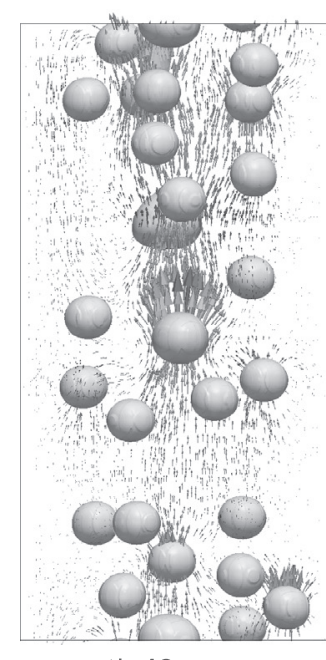

$t^{*}=12$

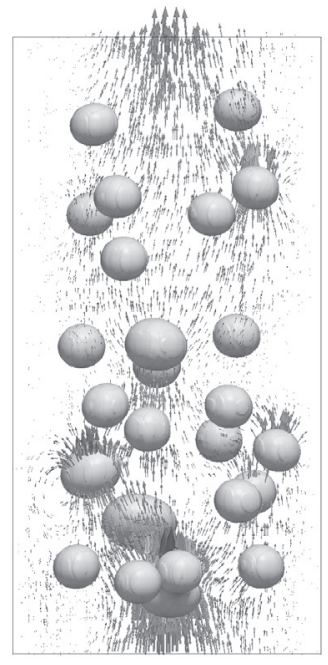

$t^{*}=24$

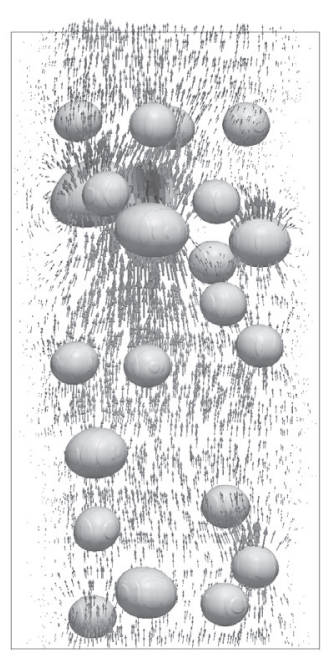

$t^{*}=38$

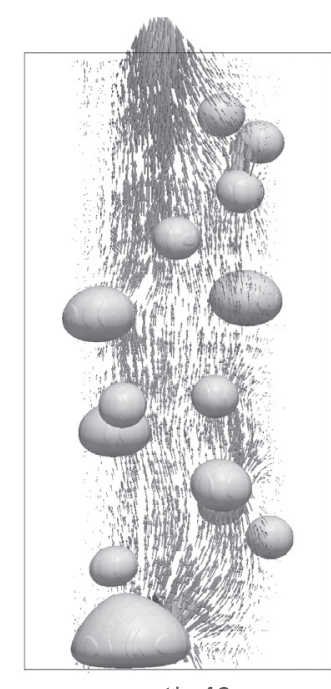

$t^{*}=46$

Fig. 22. Bubble swarm in nearly spherical shape regime; $E o=2.25, M=5.9 \times 10^{-4}, \eta_{\rho}=10, \eta_{\mu}=10$. Velocity field.

\section{Conclusions}

In the present work, a conservative level-set method has been used to study the buoyancy-driven motion of single and multiple bubbles on a one-directional periodic domain. The numerical method offers a high degree of accuracy in the prediction of terminal Reynolds numbers, drag coefficients, wake patterns and bubble shapes for a wide range of Eo and $M$ numbers. Moreover, the numerical predictions of the CLS method agree fairly well with experimental and numerical results from the literature. Numerical results for the interaction of two spherical and ellipsoidal bubbles are consistent with previous simulations performed by other numerical methodologies. For the conditions selected in this paper $\left(s=1.5 d, 0^{\circ} \leqslant \theta \leqslant 90^{\circ}\right)$, both repulsive and attractive interactions were observed, which are a function of the Reynolds number and the initial configuration angle. The performed simulation with multiple bubbles in a vertical channel shows that bubbles tend to form groups, which move through the center of the channel.

\section{Uncited references}

Brereton and Korotney (1991), Gottlieb and Chi-Wang (1998), Katz and Meneveau (1996), Lu et al. (2006), Ming et al. (2010) and Sweby (1984).

\section{Acknowledgments}

This work has been financially supported by the Ministerio de Economía y Competitividad, Secretaría de Estado de Investigación, Desarrollo e Innovación, Spain (ENE2011-28699), and by Termo Fluids S.L. Néstor Balcázar acknowledges financial support in form of a doctoral scholarship of the Agencia Española de Cooperación Internacional para el Desarrollo (AECID), Spain. The authors wish to thank the Barcelona Supercomputing Center for the use of computational facilities.

\section{References}

Balcázar, N., Jofre, L., Lehmkhul, O., Castro, J., Rigola, J., 2014. A finite-volume/levelset method for simulating two-phase flows on unstructured grids. Int. J. Multiphase Flow 64, 55-72.
Balcázar, N., Lehmkhul, O., Rigola, J., Oliva, A., 2015. A multiple marker level-set method for simulation of deformable fluid particles. Int. J. Multiphase Flow. http://dx.doi.org/10.1016/j.ijmultiphaseflow.2015.04.009.

Batchelor, G.K., 1967. An Introduction to Fluid Dynamics. Cambridge University Press.

Bhaga, D., Weber, M.E., 1981. Bubbles in viscous liquids: shapes, wakes and velocities. Int. J. Multiphase Flow 33, 109-133.

Bonometti, T., Magnaudet, J., 2007. An interface-capturing method for incompressible two-phase flows. Validation and application to bubble dynamics. J. Fluid. Mech. 105, 61-85.

Brackbill, J.U., Kothe, D.B., Zemach, C., 1992. A continuum method for modeling surface tension. J. Comput. Phys. 100, 335-354.

Brereton, G., Korotney, D. 1991. Coaxial and oblique coalescence of two rising bubbles. Dynamics of Bubbles and Vortices Near a Free Surface, vol. 119. ASME New York.

Bunner, B., Tryggvason, G., 2002. Dynamics of homogeneous bubbly flows. Part 1. Rise velocity and microstructure of the bubbles. J. Fluid Mech. 466, 17-52.

Bunner, B., Tryggvason, G., 2003. Effect of bubble deformation on the properties of bubbly flows. J. Fluid Mech. 495, 77-118.

Chakraborty, I., Biswas, G., Ghoshdastidar, P.S., 2013. A coupled level-set and volume-of-fluid method for the buoyant rise of gas bubbles in liquids. Int. J. Heat Mass Transfer 58, 240-259.

Chen, L., Li, Y., Manesh, R., 1998. The coalescence of bubbles - a numerical study. In: Third International Conference on Multiphase Flow, ICMF9́8, Lyon, France.

Chen, R.H., Tian, W.X., Su, G.H., Qiu, S.Z., Ishiwatari, Y., Oka, Y., 2011. Numerical investigation on a coalescence of bubble pairs. Chem. Eng. Sci. 66, 5055-5063.

Cheng, M., Hua, J., Lou, J., 2010. Simulation of bubble-bubble interaction using a lattice Boltzmann method. Comput. Fluids 39, 260-270.

Chorin, A.J., 1968. Numerical solution of the Navier-Stokes equations. Math Comput. 22, 745-762.

Clift, R., Grace, J.R., Weber, M.E., 1978. Bubbles, Drops and Particles. Academic Press New York.

Deen, N.G., Van Sint Annaland, M., Kuipers, J.A.M., 2009. Direct numerica simulation of complex multi-fluid flows using a combined front tracking and immersed boundary method. Chem. Eng. Sci. 64, 2186-2201.

Duineveld, P.C., 1998. Bouncing and coalescence of bubble pairs rising at high Reynolds number in pure water or aqueous surfactant solutions. Appl. Sci. Res. 58, 409-439.

Esmaeeli, A., Tryggvason, G., 2005. A DNS study of the buoyant rise of bubbles at O(100) Reynolds number. Phys. Fluids 17, 093303.

Felten, F.N., Lund, T.S., 2006. Kinetic energy conservation issues associated with the collocated mesh scheme for incompressible flow. J. Comput. Phys. 215, 465484.

Gottlieb, S., Chi-Wang, S., 1998. Total variation dimishing Runge-Kutta schemes Math. Comput. 67, 73-85.

Gueyffier, D., Li, J., Nadim, A., Scardovelli, R., Zaleski, S., 1999. Volume-of-fluid interface tracking with smoothed surface stress methods for three-dimensional flows. J. Comput. Phys. 152, 423-456.

Gupta, A., Kumar, R., 2008. Lattice Boltzmann simulation to study multiple bubble dynamics. Int. J. Heat Mass Transfer 51, 5192-5203.

Hadamard, J.S., 1911. Mouvement permanent lent dune sphere liquide et visqueuse dans un liquide visqueux. C.R. Acad. Sci. 152, 1735.

Hallez, Y., Legendre, D., 2011. Interaction between two spherical bubbles rising in a viscous liquid. J. Fluid Mech. 673, 406-431. 
Harmathy, T.Z., 1960. Velocity of large drops and bubbles in media of infinite or restricted extend. AIChE J. 6, 281-288.

Hasan, N., Zakaria, Z.B., 2011. Computational approach for a pair of bubble coalescence process. Int. J. Heat Fluid Flow 32, 755-761.

Hirt, C., Nichols, B., 1981. Volume of fluid (VOF) method for the dynamics of free boundary. J. Comput. Phys. 39, 201-225.

Hnat, J.G., Buckmaster, J.D., 1976. Spherical cap bubbles and skirt formation. Phys. Fluids 19, 182-194.

Hua, J., Stene, J., Lin, P., 2008. Numerical simulation of 3D bubbles rising in viscous liquids using a front tracking method. J. Comput. Phys. 227, 3358-3382.

Joseph, D., 2003. Rise velocity of a spherical cap bubble. J. Fluid Mech. 488, 213-223.

Katz, J., Meneveau, C., 1996. Wake induced relative motion of bubbles rising in line. Int. J. Multiphase Flow 22 (2), 239-258.

Kothe, D.B., Rider, W.J., Mosso, S.J., Brock, J.S., 1996. Volume Tracking of Interfaces Having Surface Tension in Two and Three Dimensions. AIAA 96-0859. 25

Legendre, D., Magnaudet, J., 2003. Hydrodynamic interactions between two spherical bubbles rising side by side in a viscous liquid. J. Fluid Mech. 497, 133-166.

Lehmkuhl, O., Perez-Segarra, C.D., Soria, M., Oliva, A., 2007, A new parallel unstructured CFD code for the simulation of turbulent industrial problems on low cost PC cluster. In: Proceedings of the Parallel CFD 2007 Conference, pp. 18.

Liu, H., Valocchi, A., 2012. Three-dimensional lattice Boltzmann model for immiscible two-phase flow simulations. Phys. Rev. E 85, 046309.

Lu, J., Biswas, S., Tryggvason, G., 2006. A DNS study of laminar bubbly flows in a vertical channel. Int. J. Multiphase Flow 32, 643-660.

Martínez-Mercado, J., Palacios-Morales, C.A., Zenit, R., 2007. Measurement of pseudoturbulence intensity in monodispersed bubbly liquids for $10<R e<500$. Phys. Fluids 19, 103302.

Ming, C., Jingong, H., Jing, L., 2010. Simulation of bubble-bubble interaction using a lattice Boltzmann method. Comput. Fluids 19, 260-270.

Mudde, R., 2005. Gravity-driven bubbly flows. Annu. Rev. Fluid Mech. 37, 393-423.

Mukundakrishnan, K., Quan, S., Eckmann, D.M., Ayyaswamy, P.S., 2007. Numerical study of wall effects on buoyant gas-bubble rise in a liquid-filled finite cylinder. Phys. Rev. E 76, 036308-01-036308-15.

Ohta, M., Sussman, M., 2012. The buoyancy-driven motion of a single skirted bubble or drop rising through a viscous liquid. Phys. Fluids 24, 112101.

Ohta, M., Yamaguchi, S., Yoshida, Y., Sussman, M., 2010. The sensitivity of drop motion due to the density and viscosity ratio. Phys. Fluids $22,072102$.

Olsson, E., Kreiss, G., 2005. A conservative level set method for two phase flow. J. Comput. Phys. 210, 225-246.

Osher, S., Sethian, J.A., 1988. Fronts propagating with curvature-dependent speed: algorithms based on Hamilton-Jacobi formulations. J. Comput. Phys. 79, 175210.

Peskin, C.S., 1977. Numerical analysis of blood flow in the heart. J. Comput. Phys. 25 $220-252$.

Pournader, O., Mortazavi, S., 2013. Three dimensional interaction of two drops driven by buoyancy. Comput. Fluids $88,543-556$

Rhie, C.M., Chow, W.L., 1983. Numerical study of the turbulent flow past an airfoil with trailing edge separation. AIAA J. 21, 1525-1532.

Rodrigue, D., 2001. Generalized correlation for bubble motion. AIChE J. 47, 39-44.

Rybczynski, W., 1911. Uber die fortschreitende bewegung einer flssigen kugel in einem zhen medium. Bull. Acad. Sci. Cracovi, Ser. A, 40
Ryskin, G., Leal, L.G., 1984. Numerical solution of free-boundary problems in fluid mechanics. Part 2. Buoyancy-driven motion of a gas bubble through a quiescent liquid. J. Fluids Mech. 148, 19-35.

Sanada, T., Sato, A., Shirota, M., Watanabe, M., 2009. Motion and coalescence of a pair of bubbles rising side by side. Chem. Eng. Sci. 64, 2659-2671.

Sheu, W.H., Yu, C.H., Chiu, P.H., 2009. Development of a dispersively accurate conservative level set scheme for capturing interface in two phase flows. J. Comput. Phys. 228, 661-686.

Smolianski, A., Haario, H., Luukka, P., 2008. Numerical study of dynamics of single bubbles and bubble swarms. Appl. Math. Model. 32, 641-659.

Stewart, C.W., 1995. Bubble interaction in low-viscosity liquids. Int. J. Multiphase Flow 21, 1037-1046.

Sun, D.L., Tao, W.Q., 2010. A coupled volume-of-fluid and level-set (VOSET) method for computing incompressible two-phase flows. Int. J. Heat Mass Transfer 53, 645-655.

Sussman, M., Puckett, E.G., 2000. A coupled level set and volume-of-fluid method for computing 3D and axisymmetric incompressible two-phase flows. J. Comput. Phys. 162, 301-337.

Sussman, M., Smereka, P., Osher, S., 1994. A level set approach for computing solutions to incompressible two-phase flow. J. Comput. Phys. 144, 146-159.

Sweby, P.K., 1984. High resolution using flux limiters for hyperbolic conservation laws. SIAM J. Numer. Anal. 21, 995-1011.

Takemura, F., Magnaudet, J., 2003. The transverse force on clean and contaminated bubbles rising near a vertical wall at moderate Reynolds number. J. Fluid Mech. 495, 235-253.

Trias, F.X., Lehmkuhl, O., 2011. A self-adaptive strategy for the time integration of Navier-Stokes equations. Numer. Heat Transfer, B 60, 116-134.

Tryggvason, G., Bunner, B., Esmaeeli, A., Juric, D., Al-Rawahi, N., Tauber, W., Han, J., Nas, S., Jan, Y.-J., 2001. A front-tracking method for the computations of multiphase flow. J. Comput. Phys. 169, 708-759.

Tryggvason, G., Dabiri, S., Abouhasanzadeh, B., Jaicai, L., 2013. Multiscale considerations in direct numerical simulations of multiphase flows. Phys. Fluids 25, 031302

Unverdi, S., Tryggvason, G., 1992. A front-tracking method for viscous, incompressible, multifluid flows. J. Comput. Phys. 100, 25-37.

Van Sint Annaland, M., Deen, N.G., Kuipers, J.A.M., 2005. Numerical simulation of gas bubbles behaviour using a three-dimensional volume-of-fluid method. Chem. Eng. Sci. 60, 2999-3011.

Wang, Z., Yang, J., Koo, B., Stern, F., 2009. A coupled level set and volume-of-fluid method for sharp interface simulation of plunging breaking waves. Int. J. Multiphase Flow 35, 227-246.

Yin, X., Koch, L., 2008. Lattice-Boltzmann simulation of finite Reynolds number buoyancy-driven bubbly flows in periodic and wall domains. Phys. Fluids 20, 103304.

Yue, P., Feng, J.J., Bertelo, C.A., Hu, H.H., 2007. An arbitrary Lagrangian-Eulerian method for simulating bubble growth in polymer foaming. J. Comput. Phys. 226, 2229-2249.

Yu, Z., Yang, H., Fan, L., 2011. Numerical simulation of bubble interactions using adaptive lattice Boltzmann method. Chem. Eng. Sci. 66, 3441-3451.

Zenit, R., Koch, D.L., Sangani, A.S., 2001. Measurements of the average properties of a suspension of bubbles rising in a vertical channel. J. Fluid Mech. 429, 307-342. 